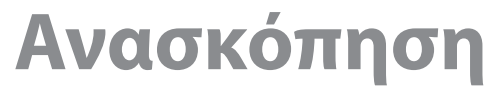 Review
}

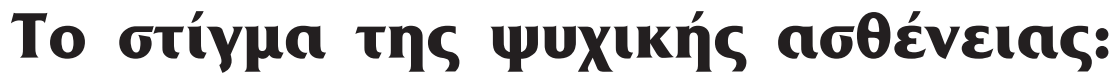

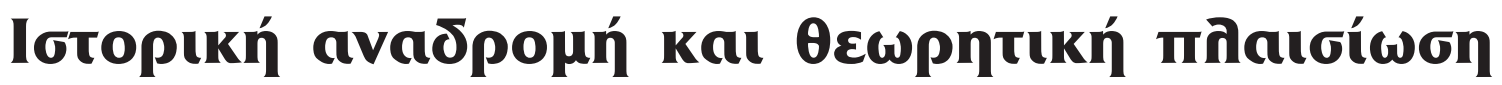

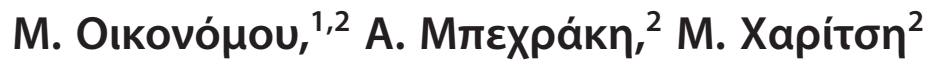

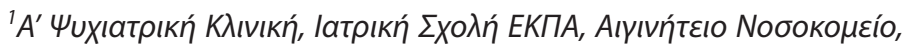

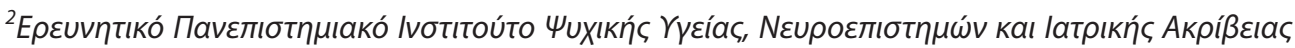

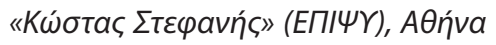

Uuxıатрıкń 2020, 31:36-46

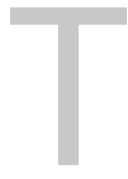

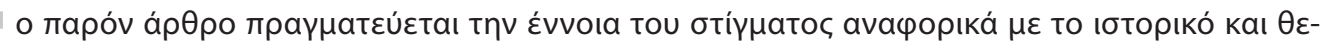

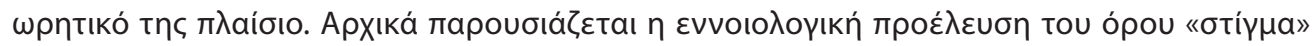

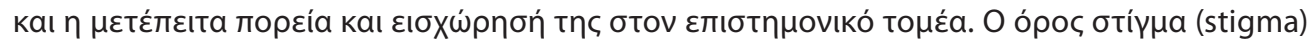

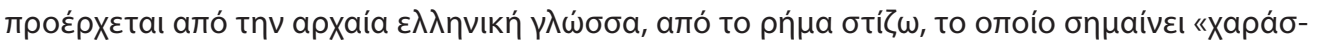

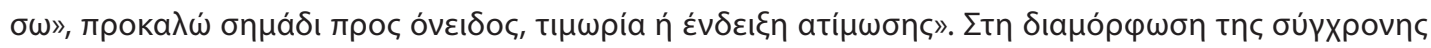

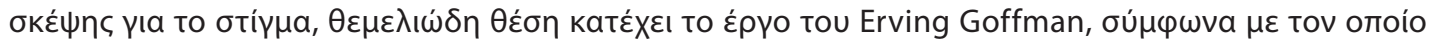

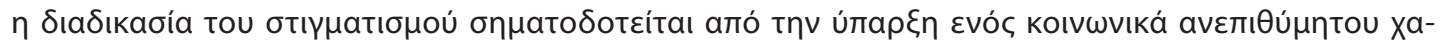

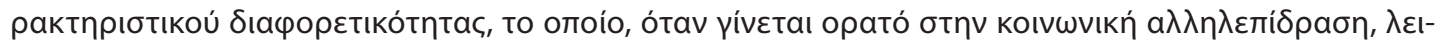

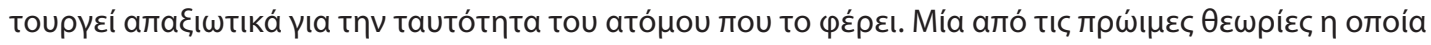

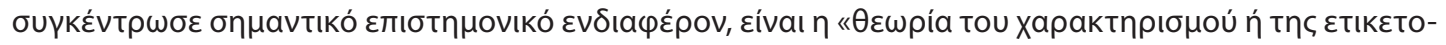

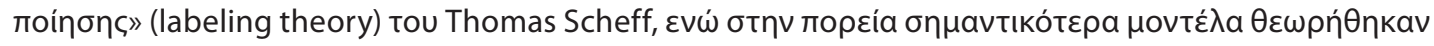

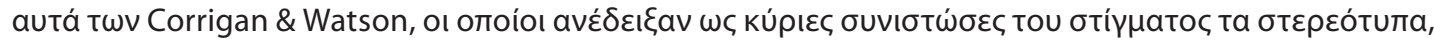

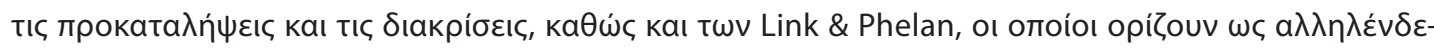

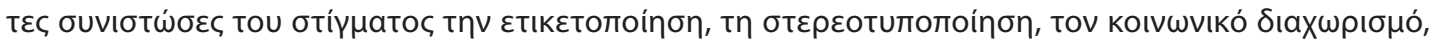

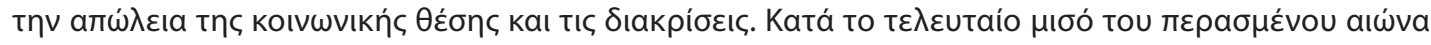

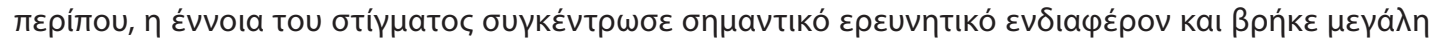

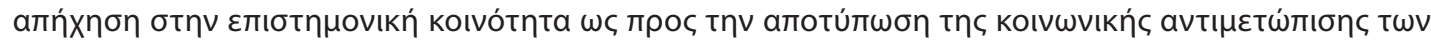

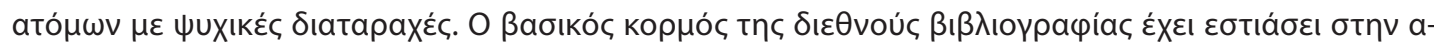

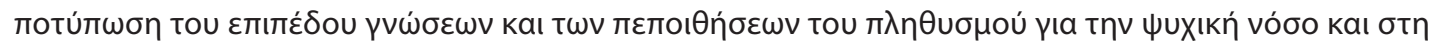

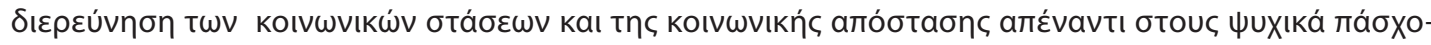

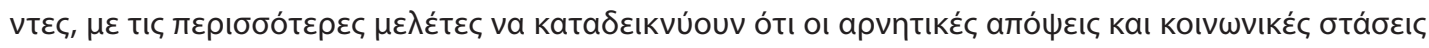




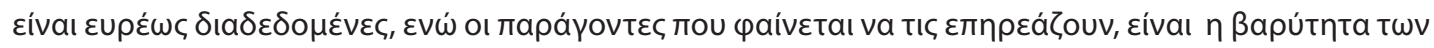

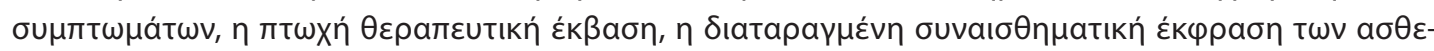

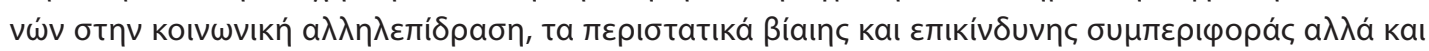

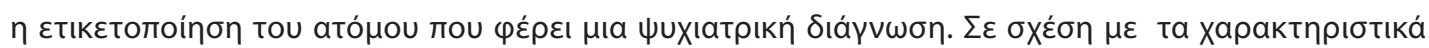

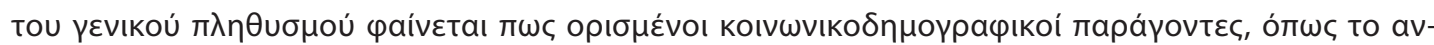

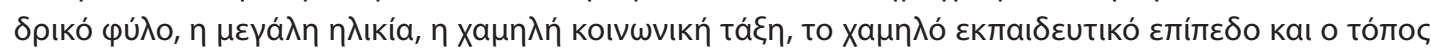

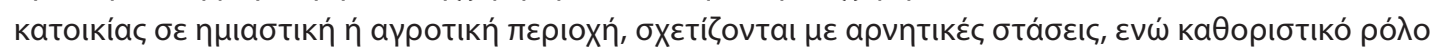

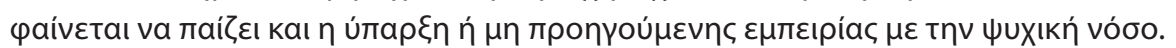

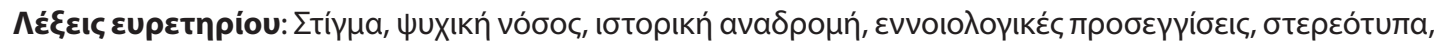

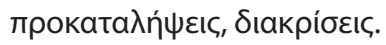

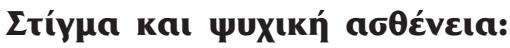

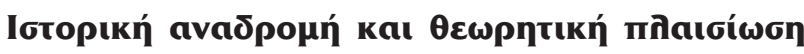

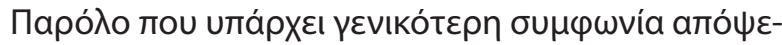

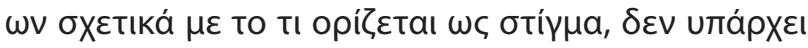

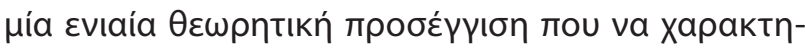

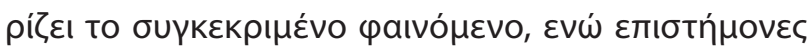

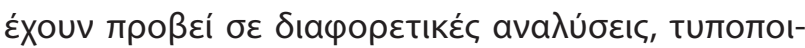

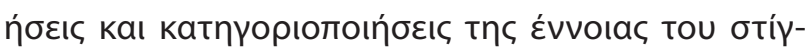

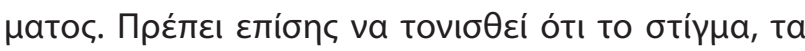

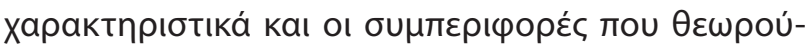

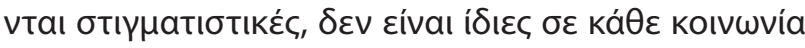

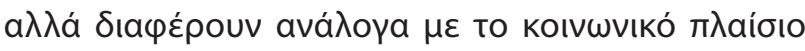

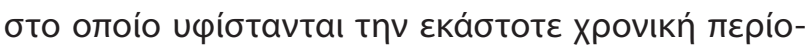

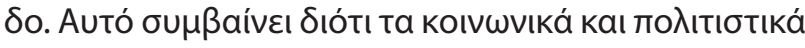

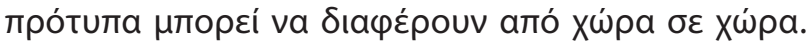

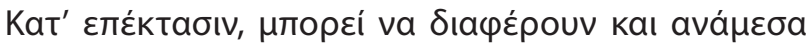

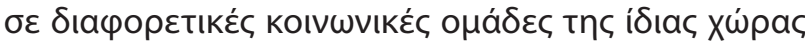

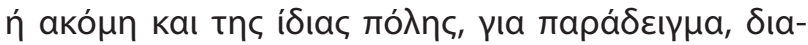

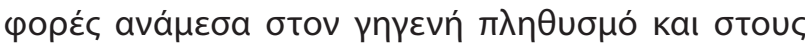

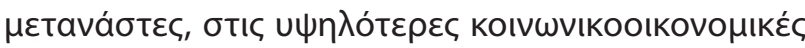

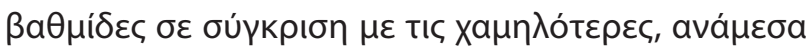

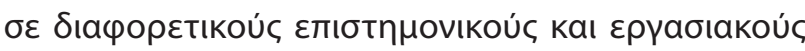

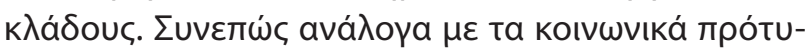

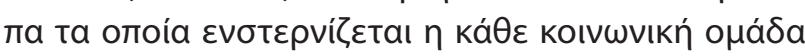

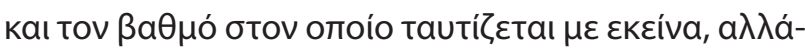

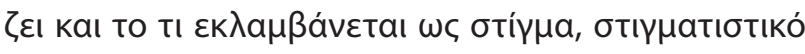

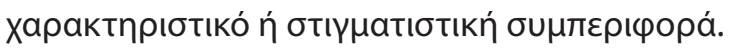

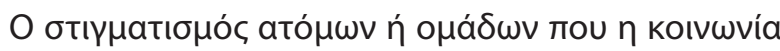

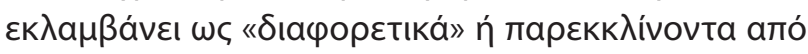

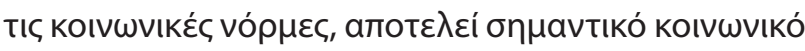

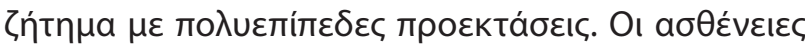

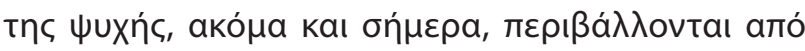

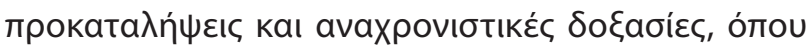

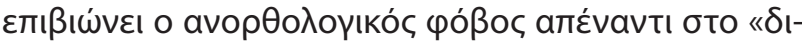

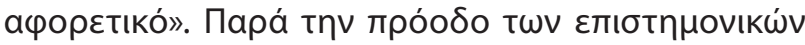

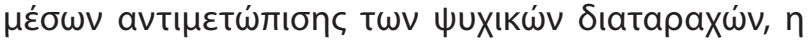

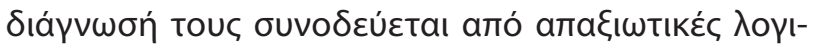

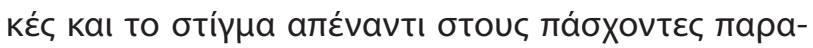

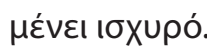

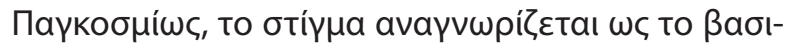

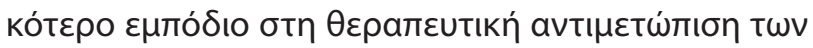

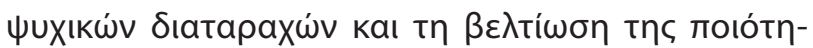

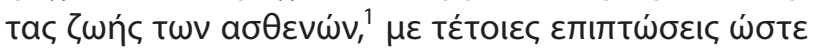

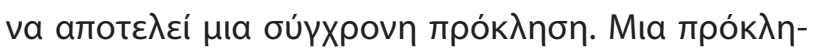

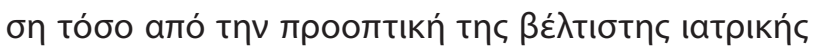

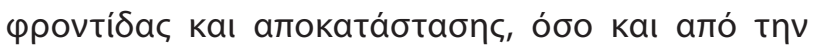

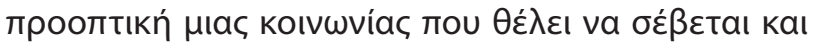

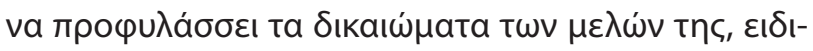

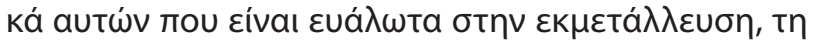

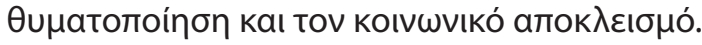

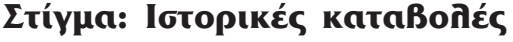

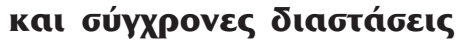

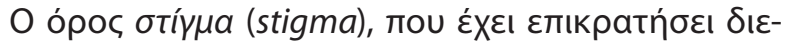

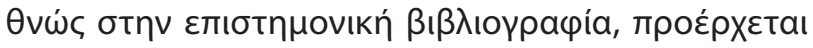

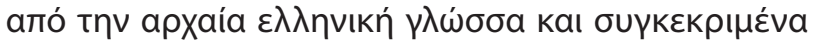

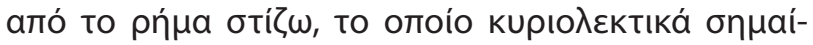

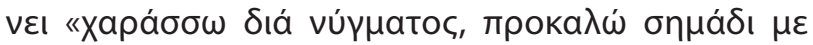

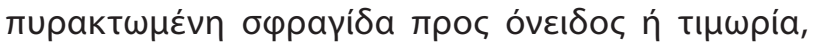

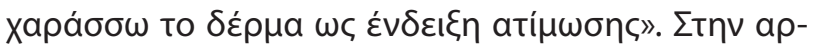

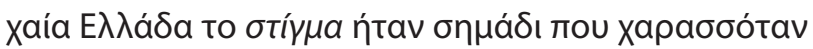

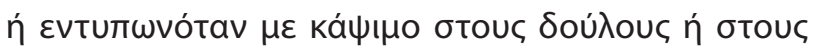

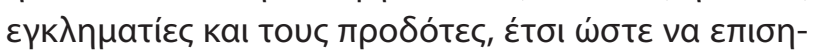

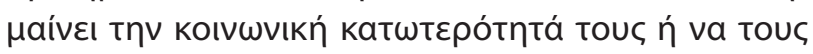

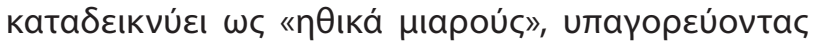

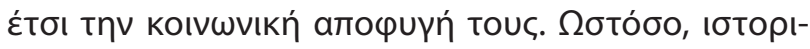

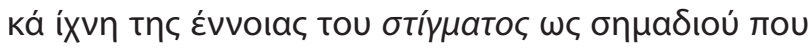

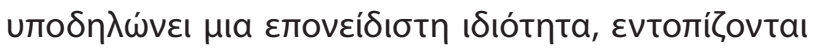

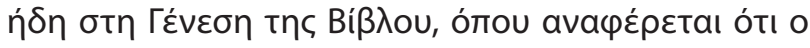

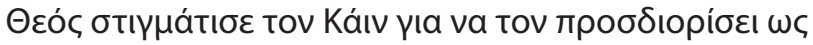




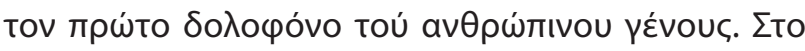

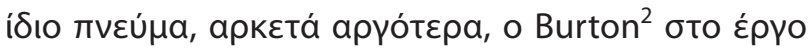

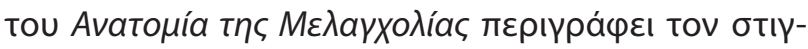

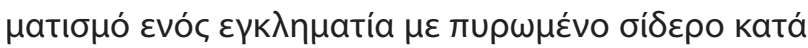

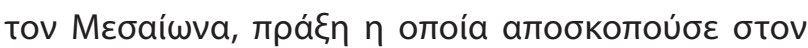

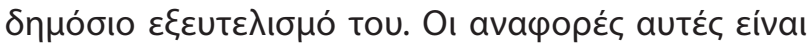

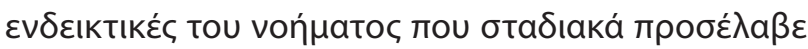

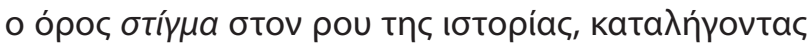

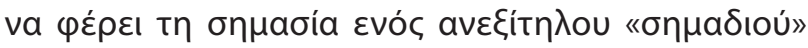

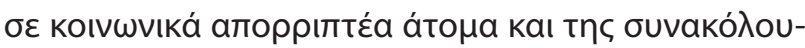

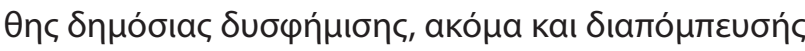
Tous.

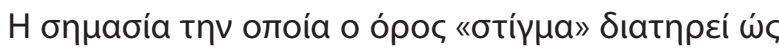

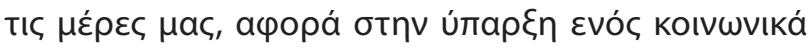

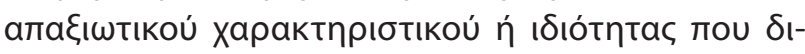

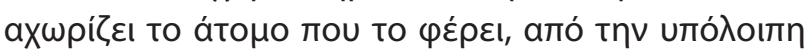

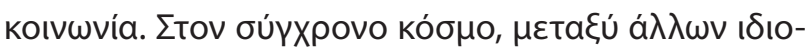

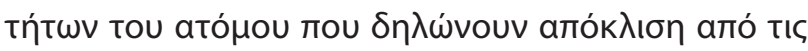

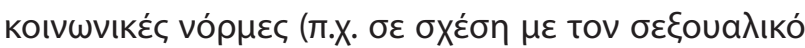

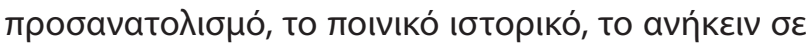

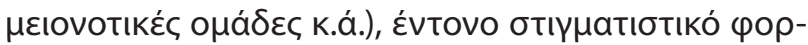

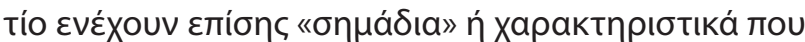

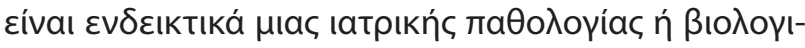

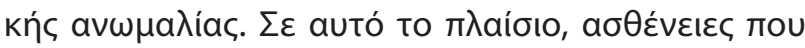

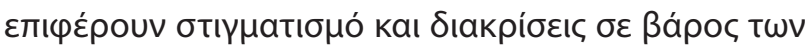

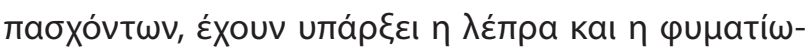

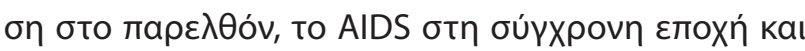

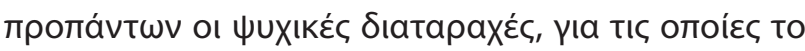

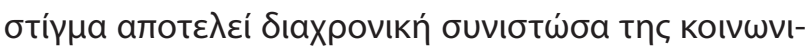

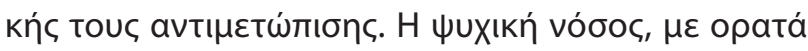

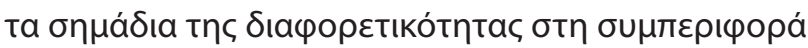

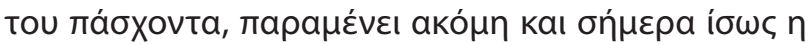

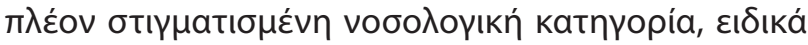

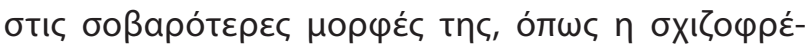
vela. ${ }^{3,4}$

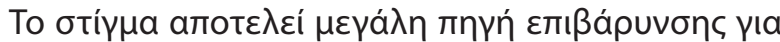

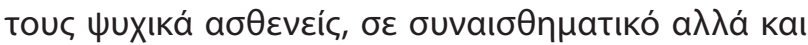

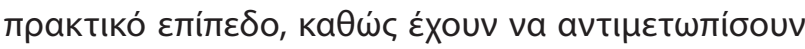

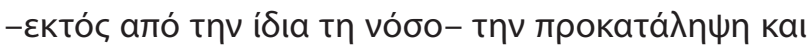

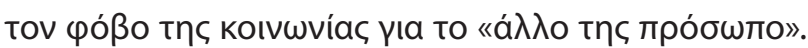

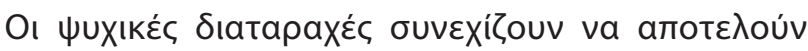

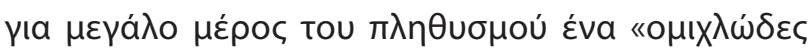

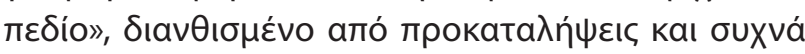

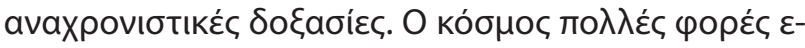

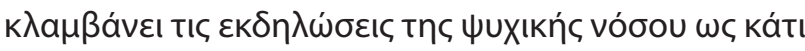

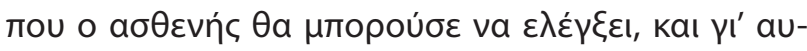

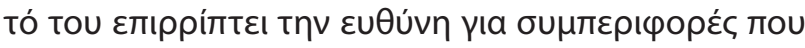

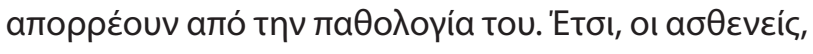

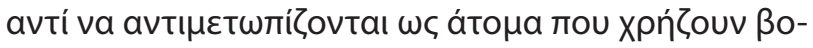

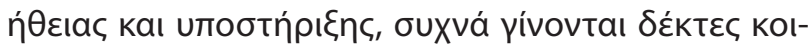

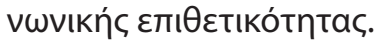

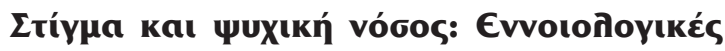

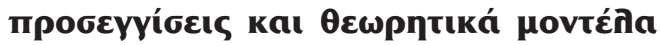

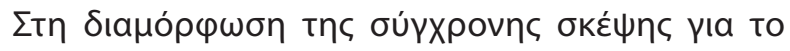

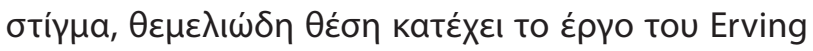

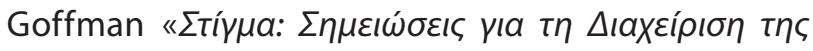

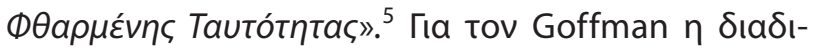

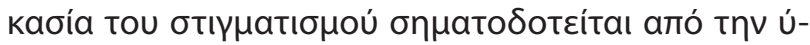

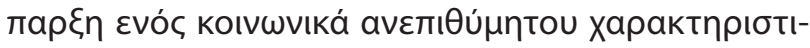

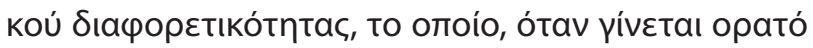

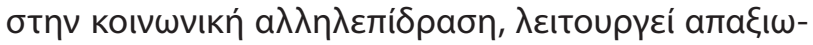

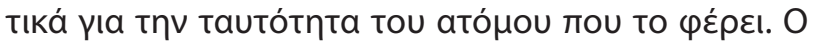

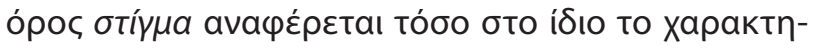

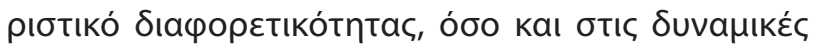

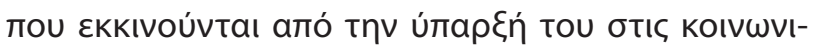

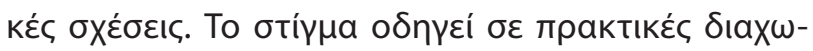

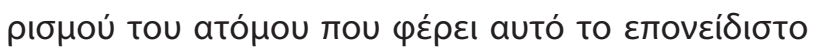

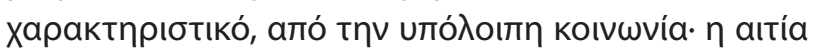

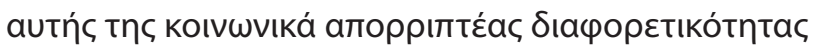

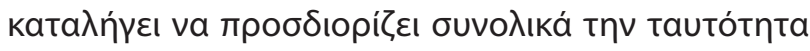

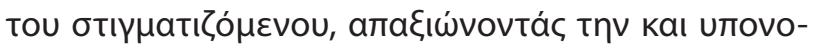

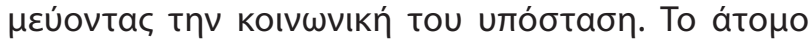

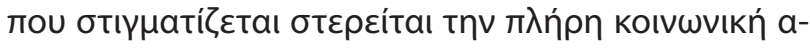

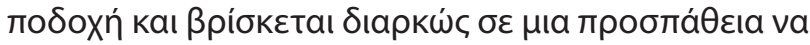

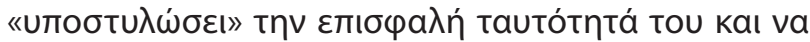

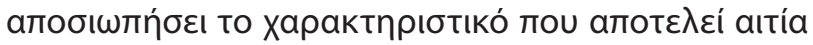

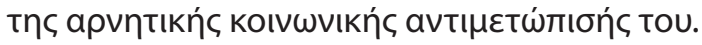

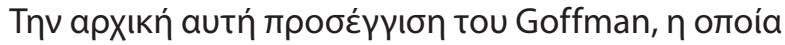

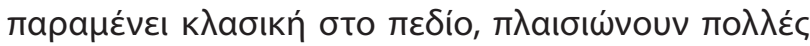

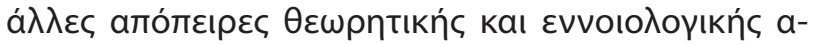

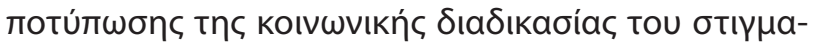

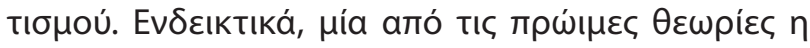

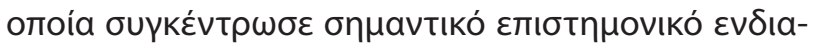

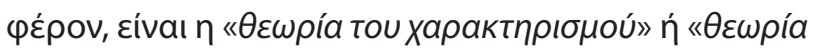

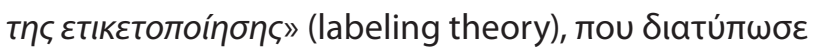
o Thomas Scheff. ${ }^{6} \Sigma u ́ \mu \varphi \omega v a \mu \varepsilon$ tn $\theta \varepsilon \omega \rho i ́ a$ autń, $\eta$

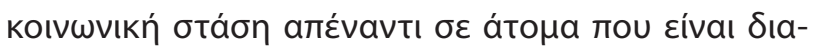

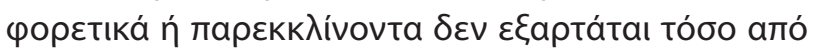

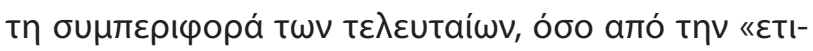

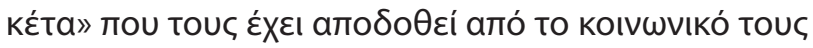

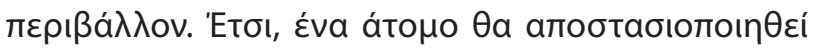

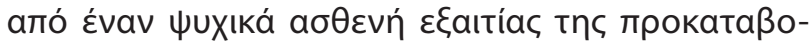

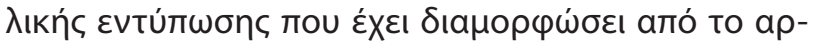




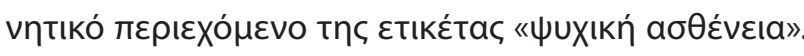

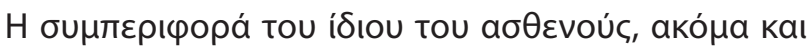

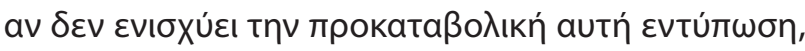

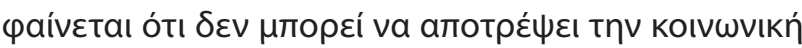

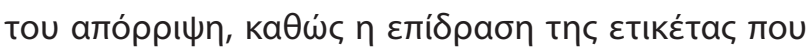

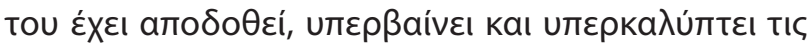

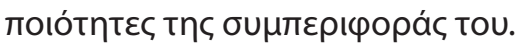

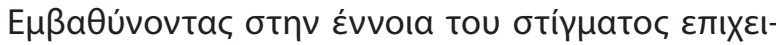

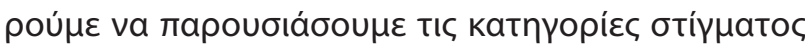

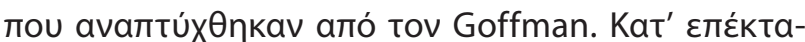

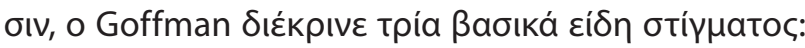

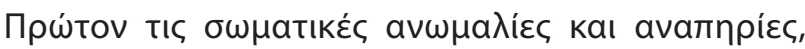

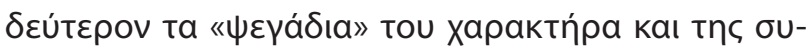

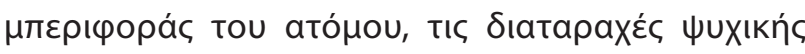

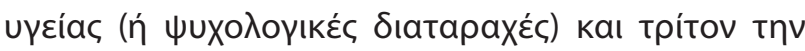

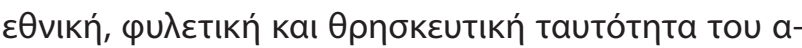

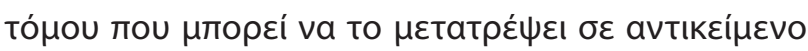

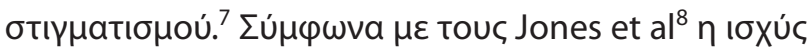

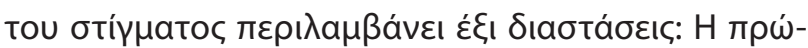

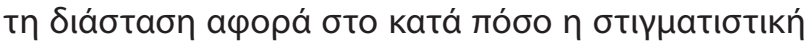

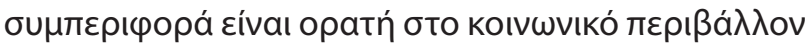

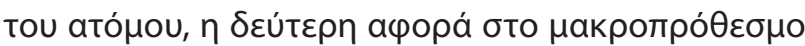

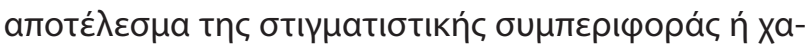

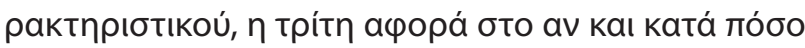

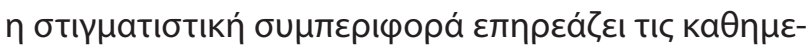

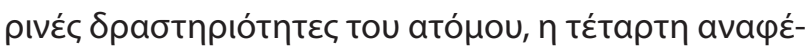

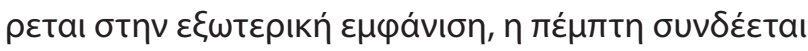

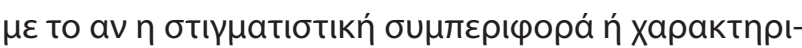

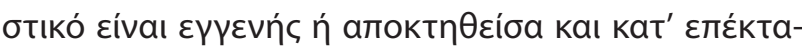

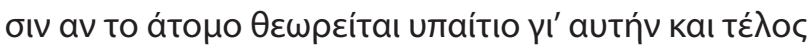

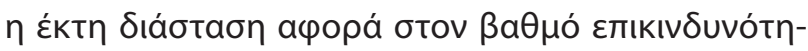

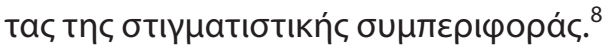

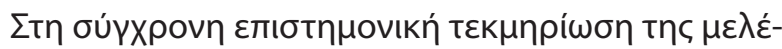

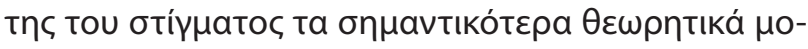

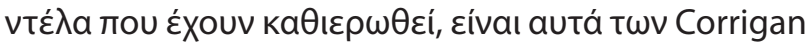
\& Watson ${ }^{9}$ kal $T \omega v$ Link \& Phelan. ${ }^{10}$ Oı Corrigan et

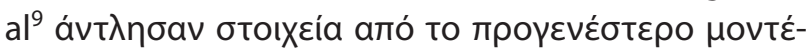

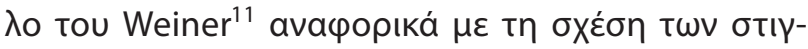

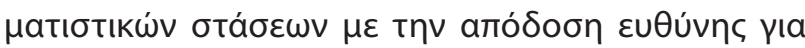

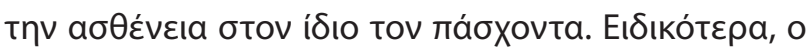

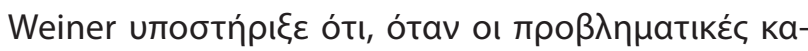

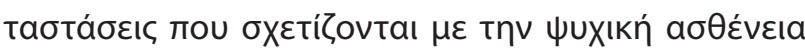

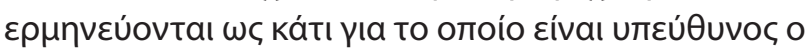

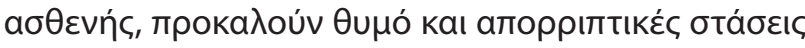

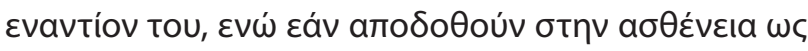

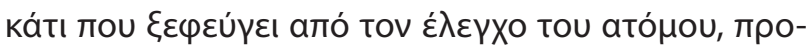

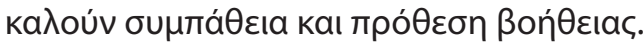

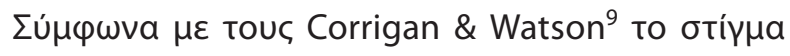

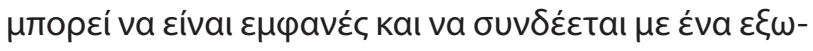

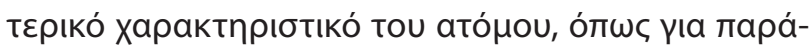

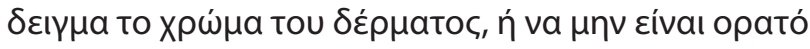

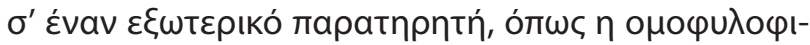

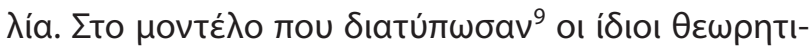

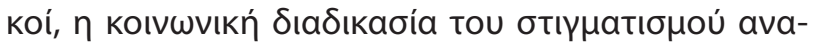

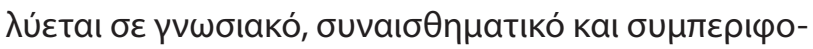

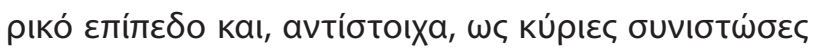

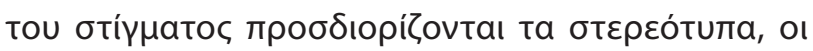

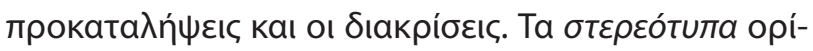

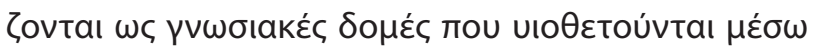

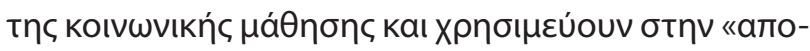

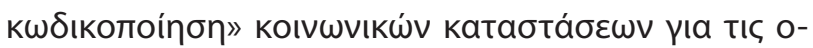

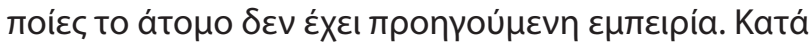

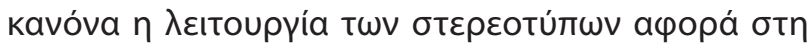

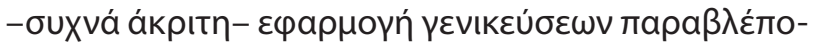

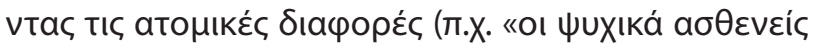

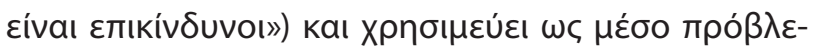

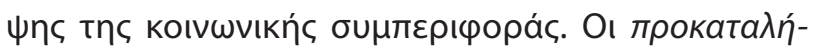

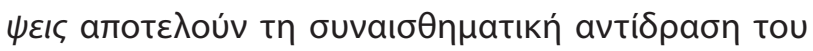

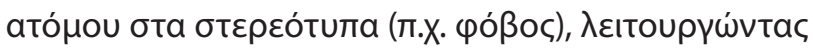

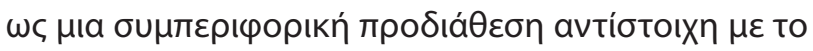

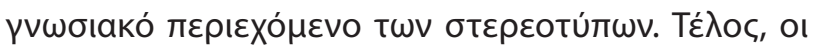

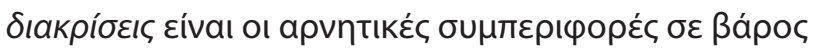

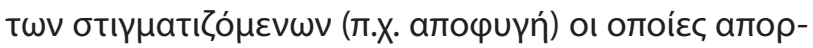

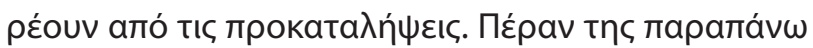

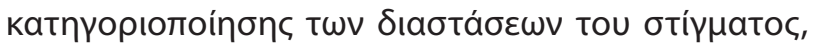

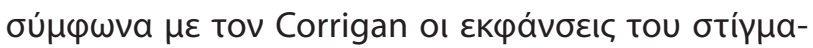

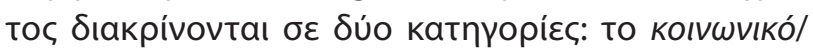

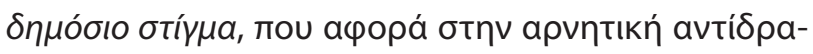

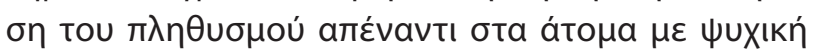

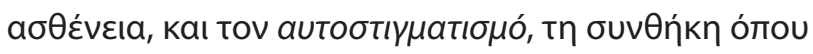

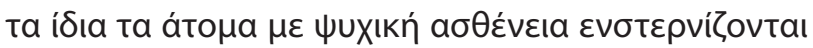

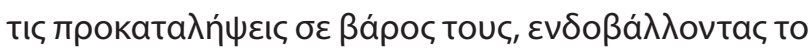

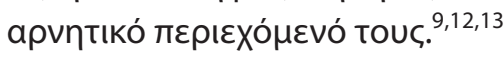

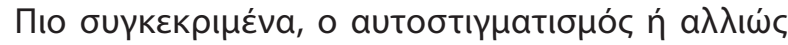

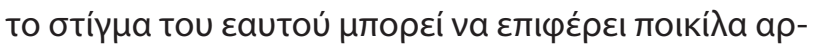

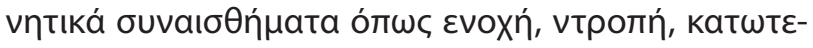

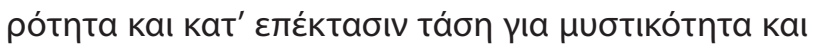

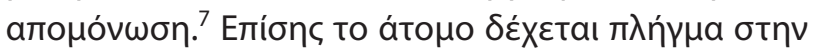

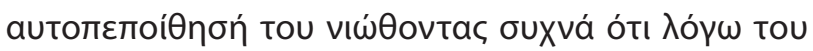

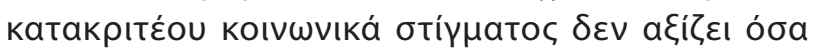

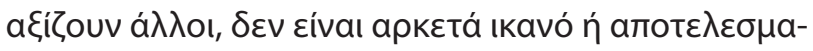

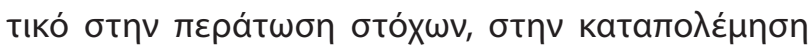

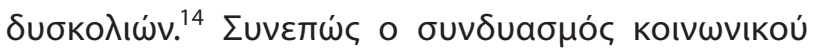

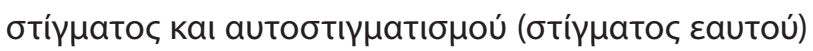




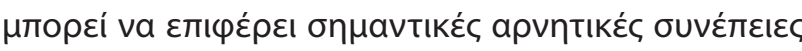

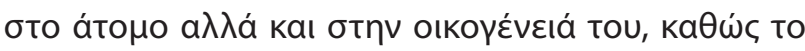

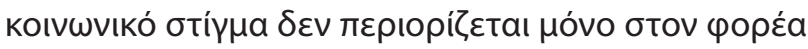

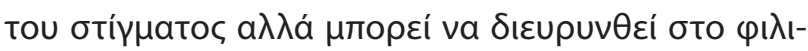

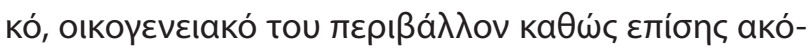

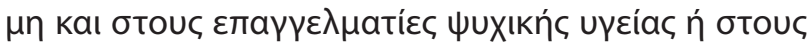

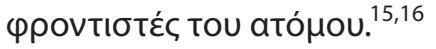

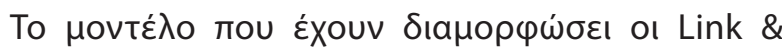

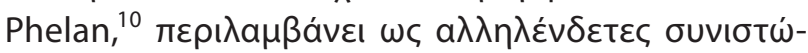

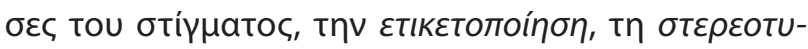

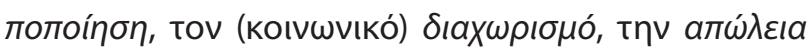

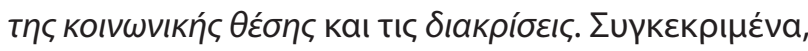

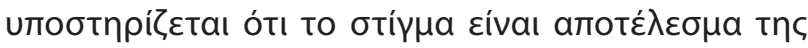

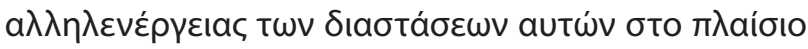

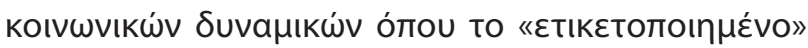

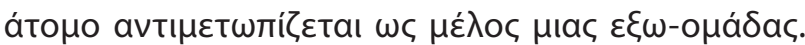

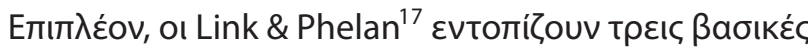

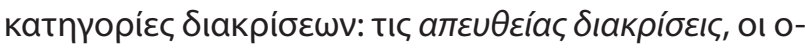

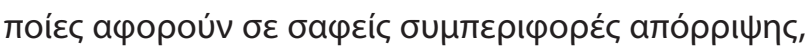

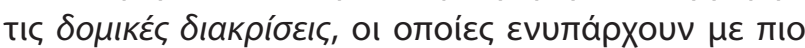

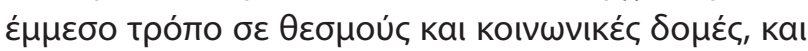

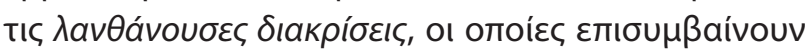

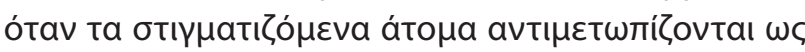

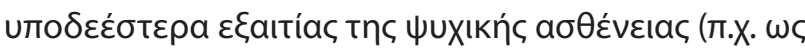

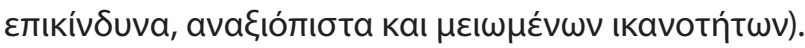

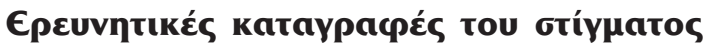

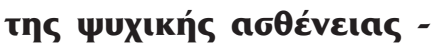

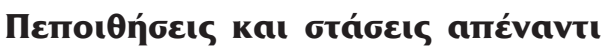

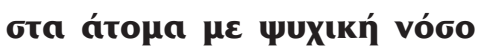

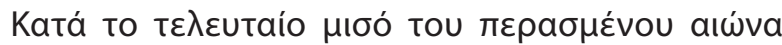

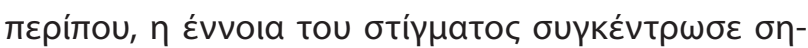

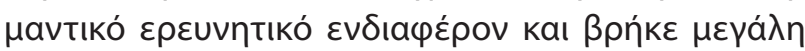
a

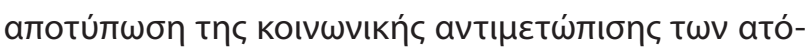

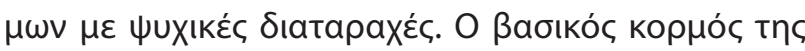

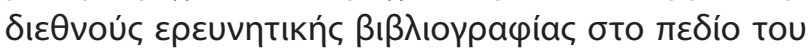

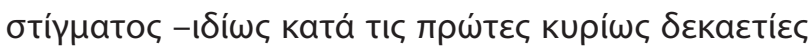

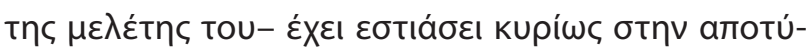

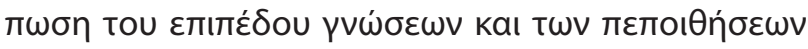

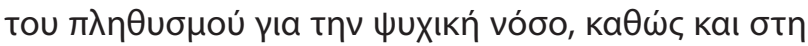

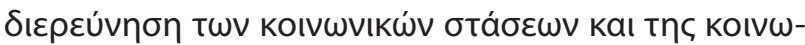

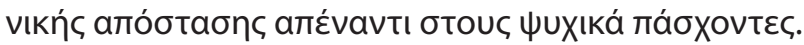

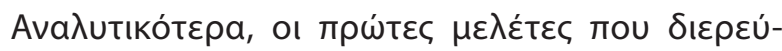

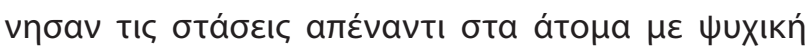

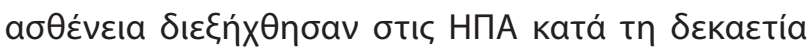

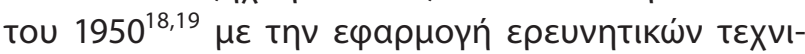

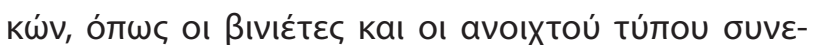

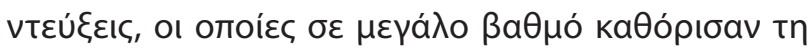

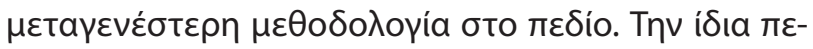

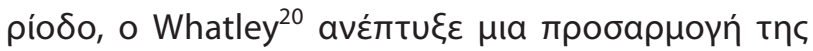

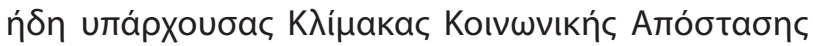

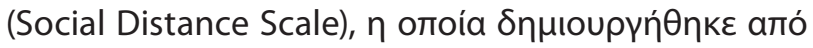

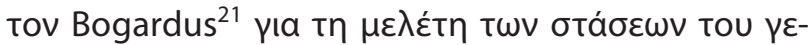

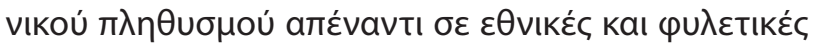

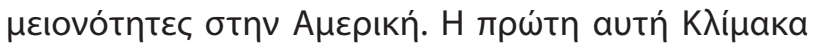

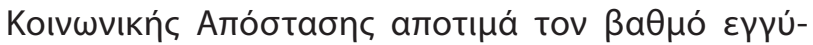

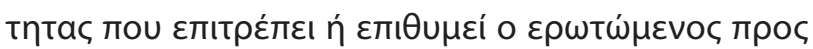

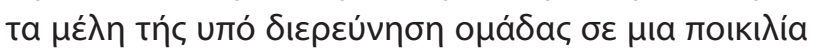

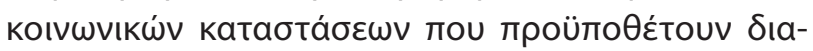

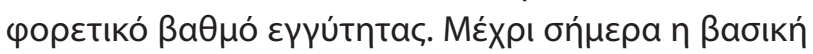

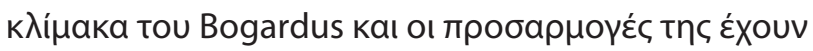

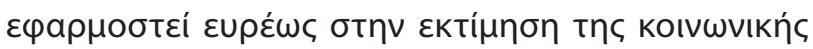

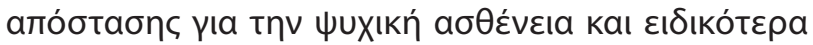

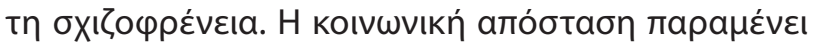

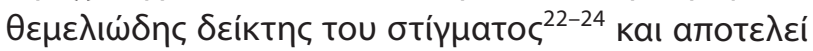

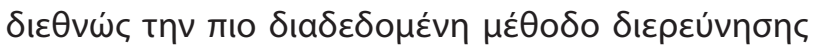

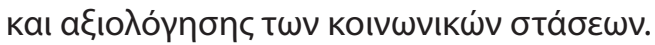

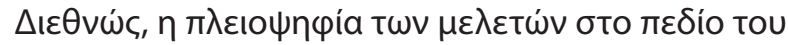

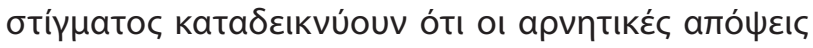

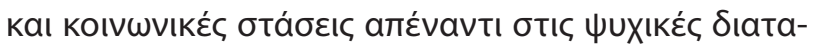

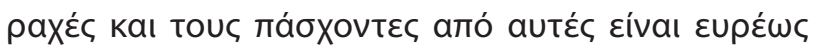

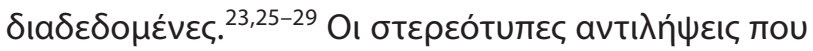

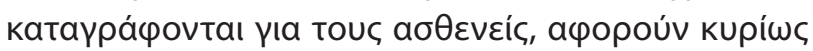

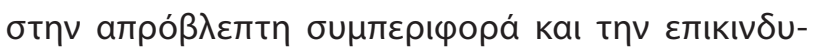

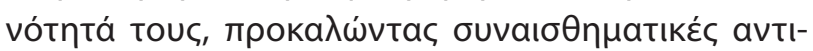

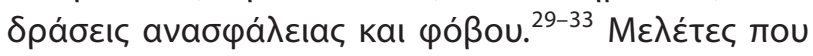

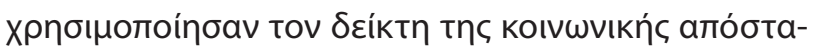

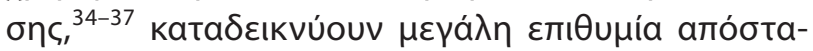

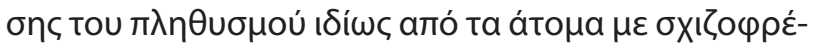

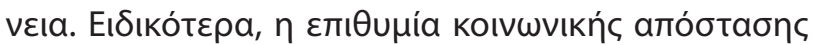

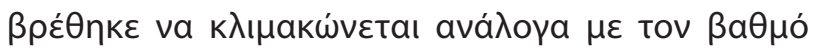

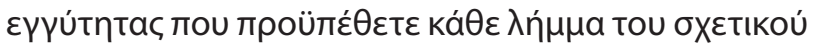

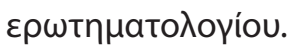

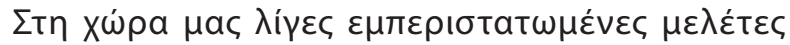

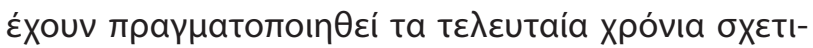

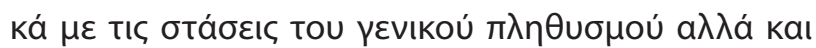

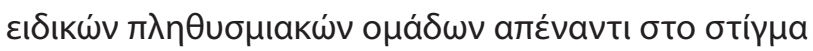

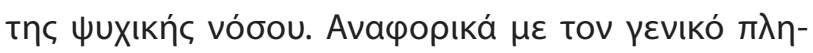

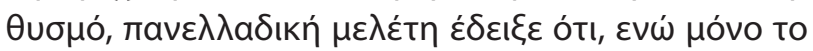

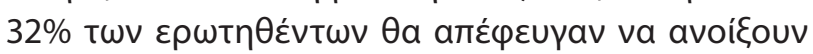

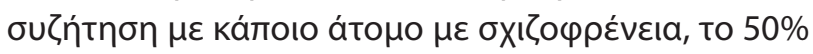

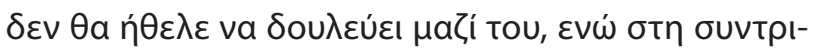

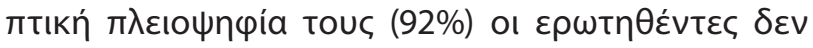




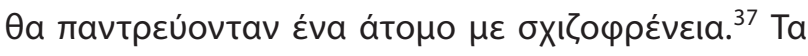

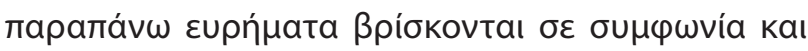

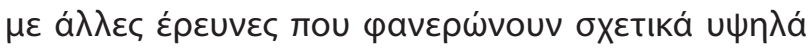

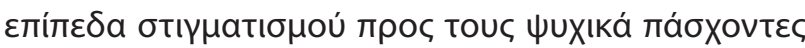

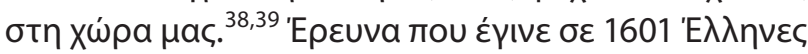

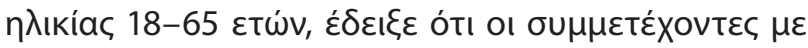

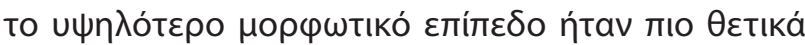

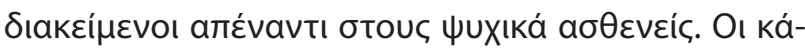

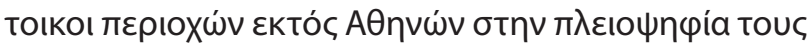

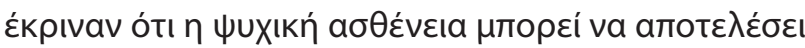

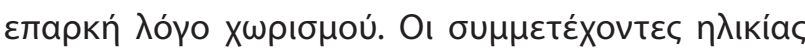

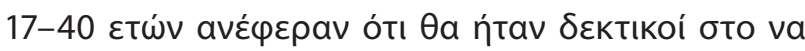

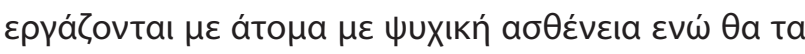

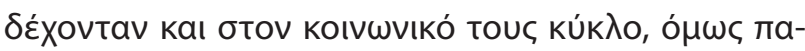

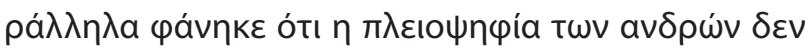

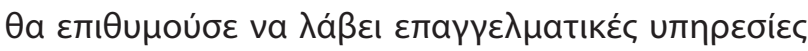

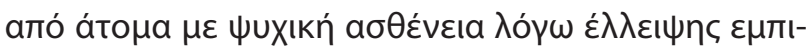

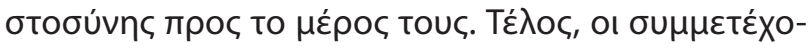

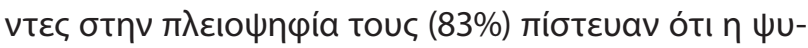

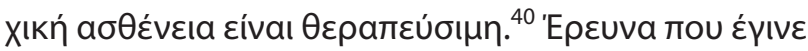

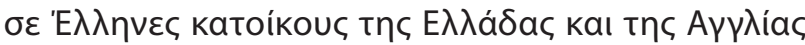

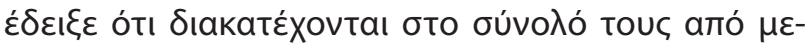

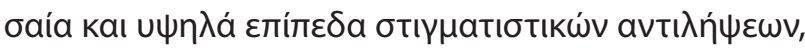

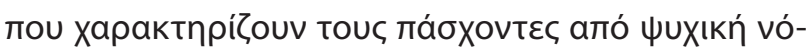

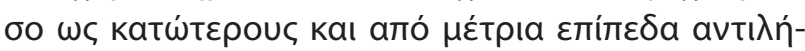

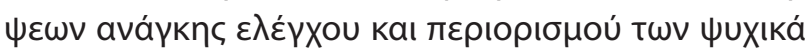

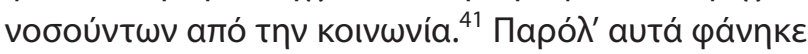

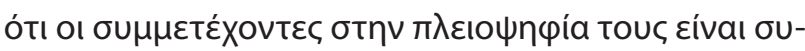

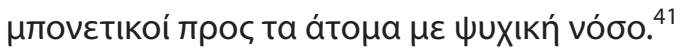

'Oбov a

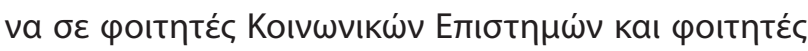

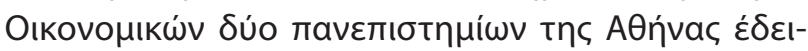

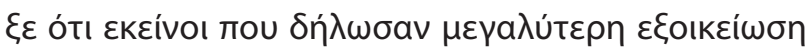

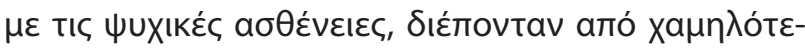

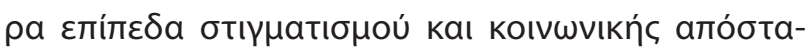

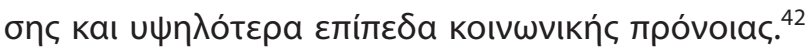

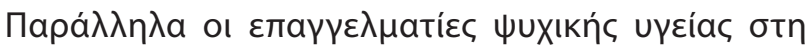

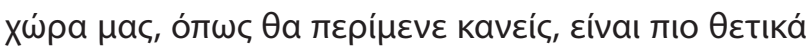

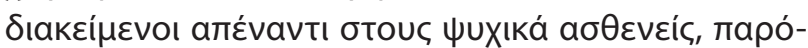

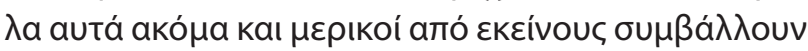

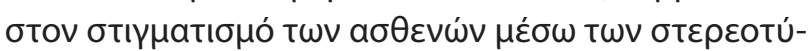

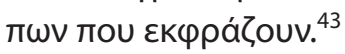

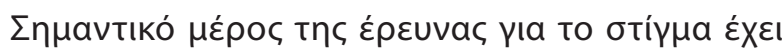

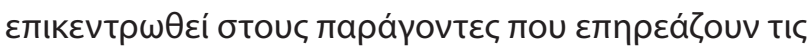

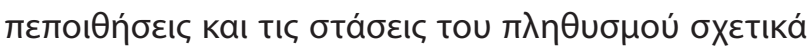

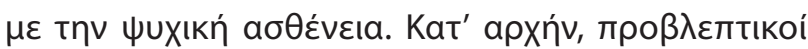

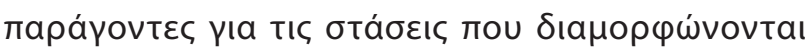

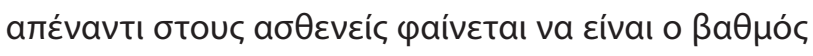

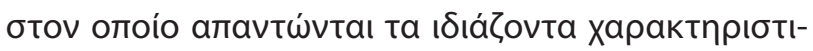

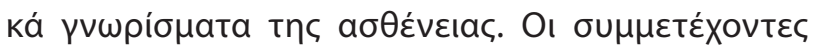

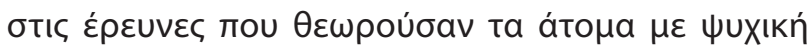

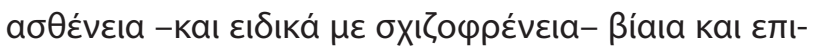

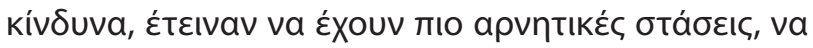

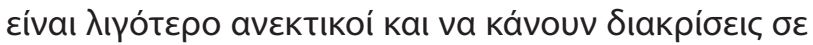

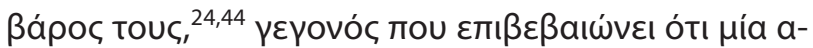

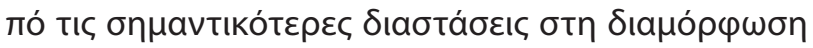

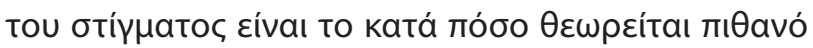

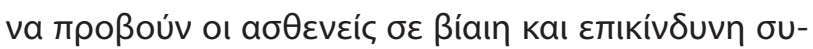
нтвріфора́.

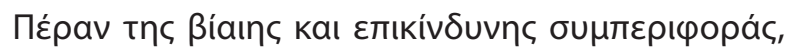

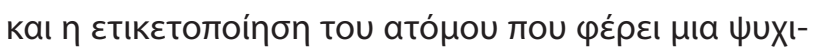

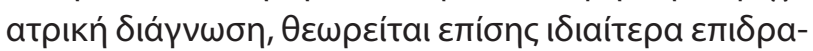

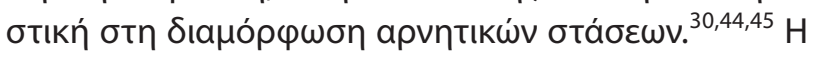

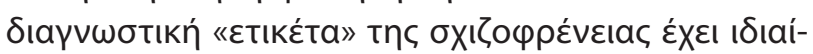

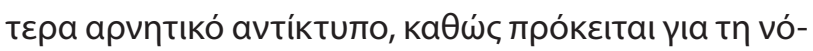

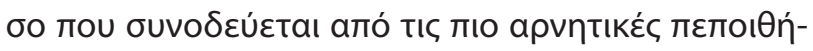

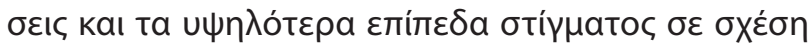

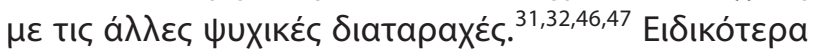

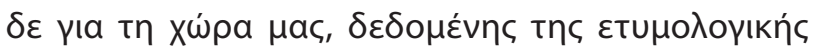

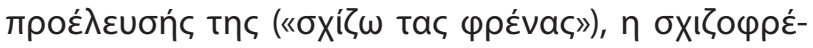

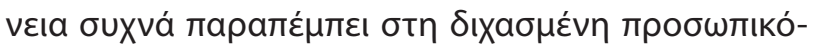

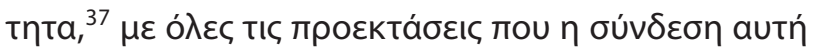

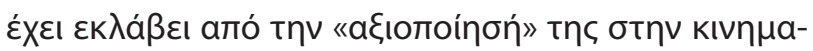

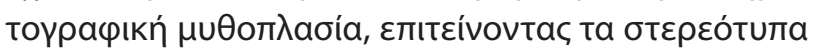

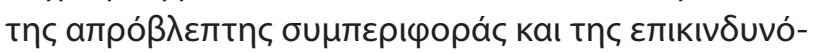

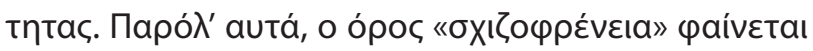

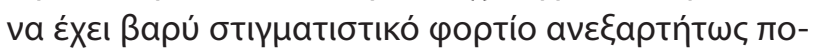

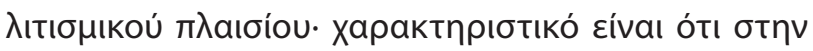

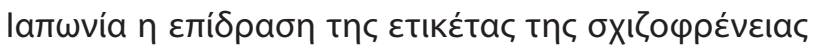

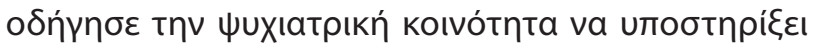

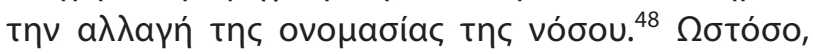

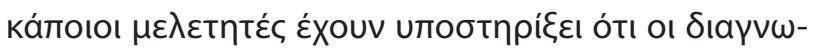

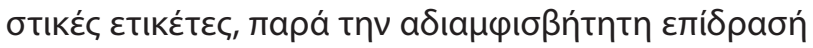

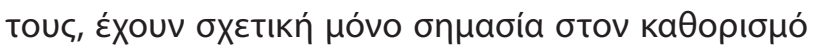

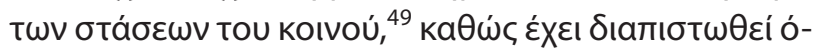

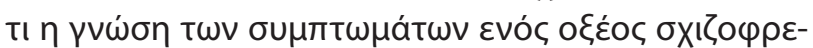

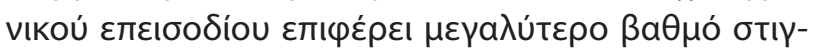

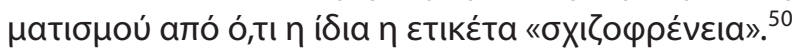

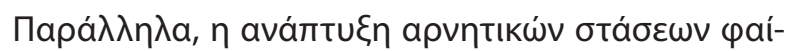

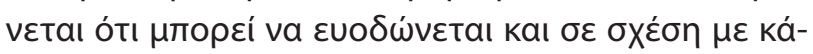

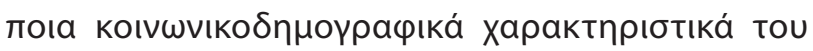

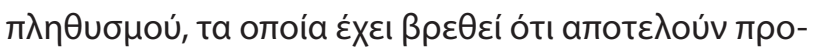

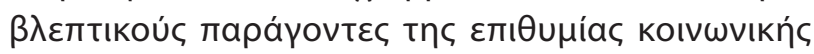

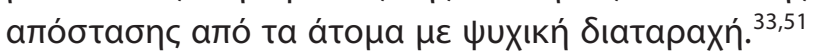




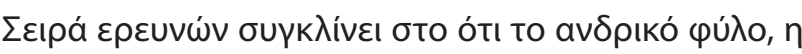

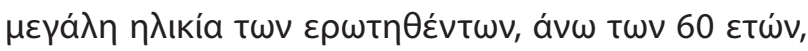

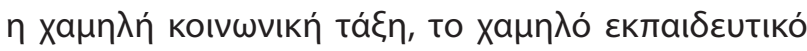

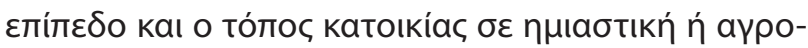

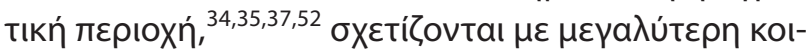

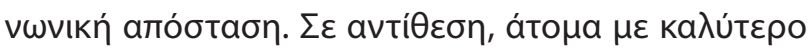

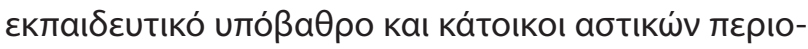

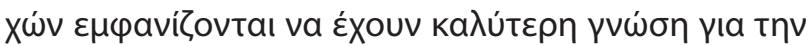

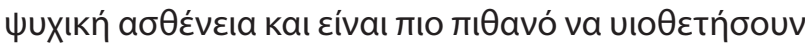

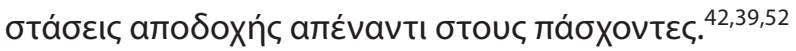

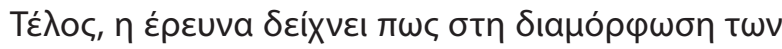

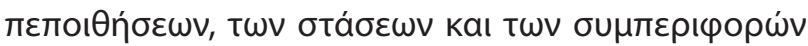

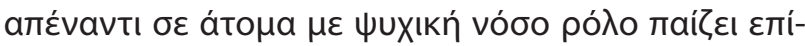

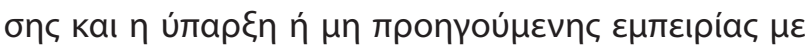

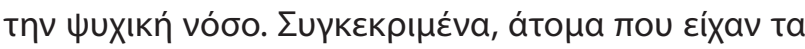

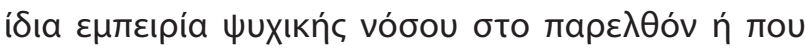

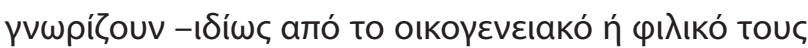

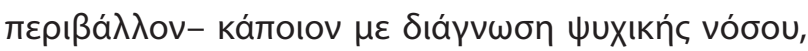

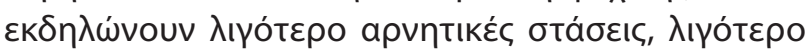

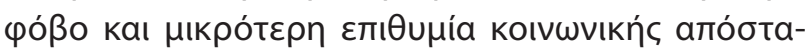

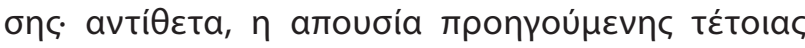

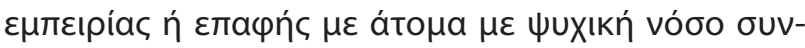

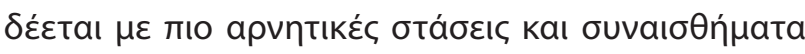

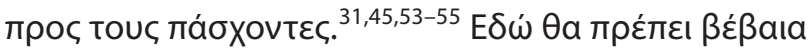

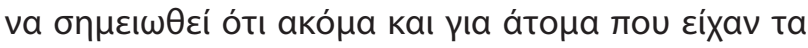

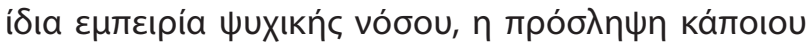

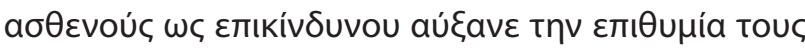

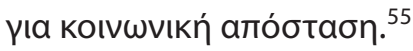

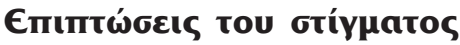

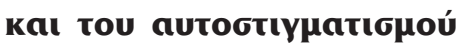

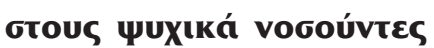

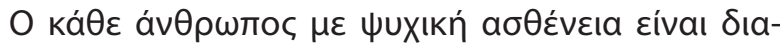

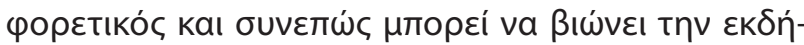

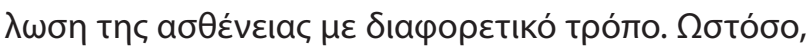

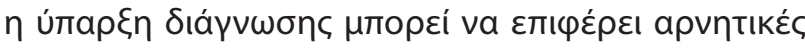

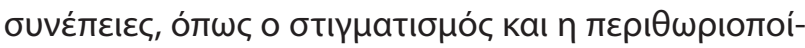

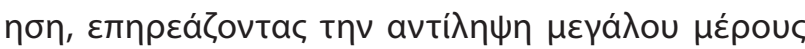

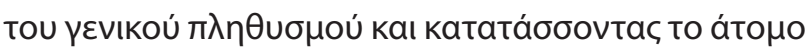

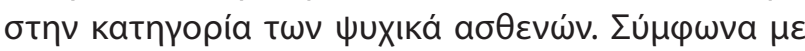

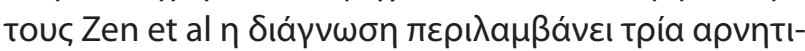

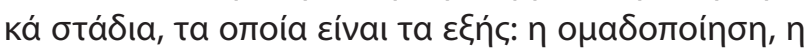

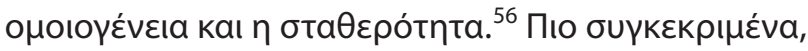

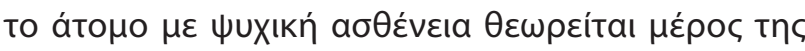

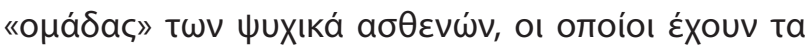

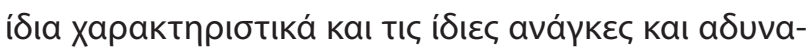

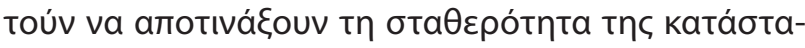

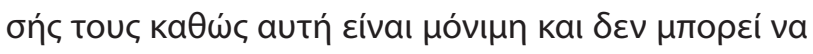

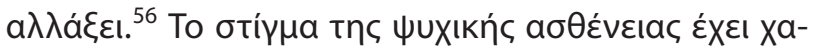

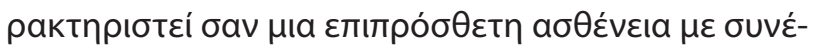

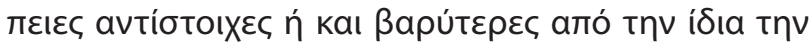

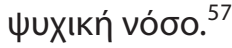

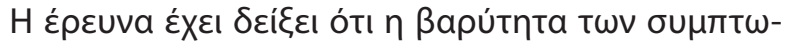

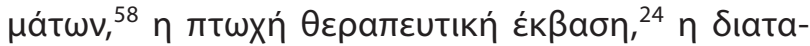

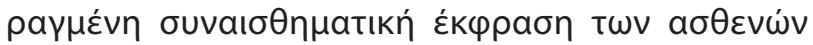

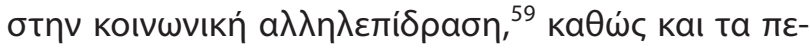

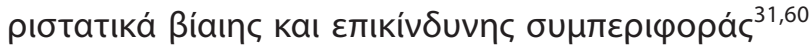

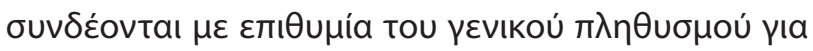

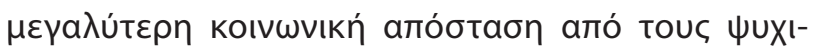

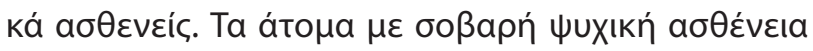

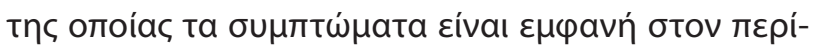

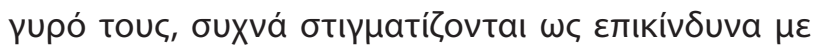

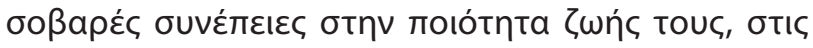

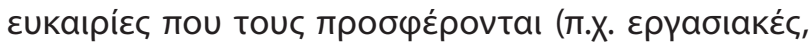

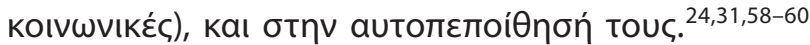

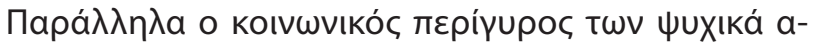

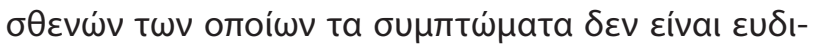

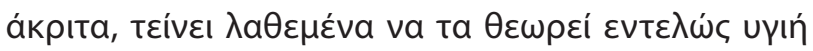

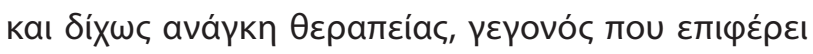

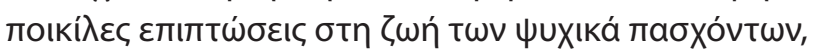

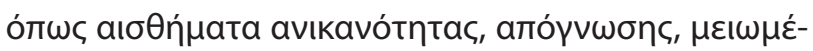

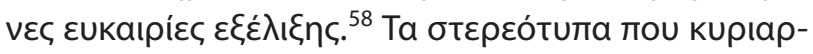

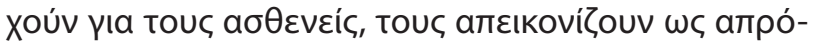

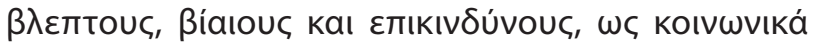

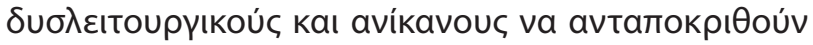

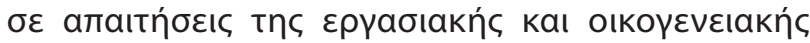

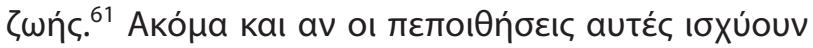

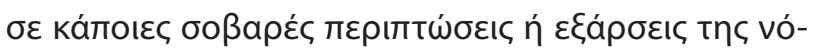

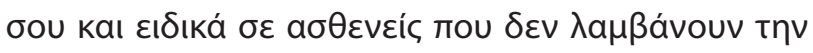

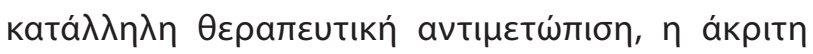

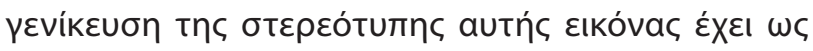

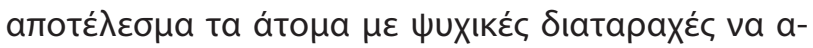

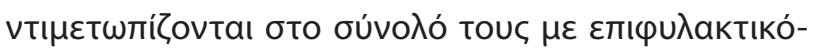

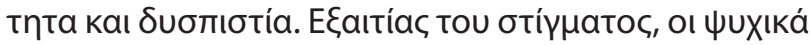

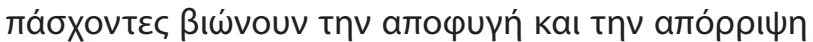

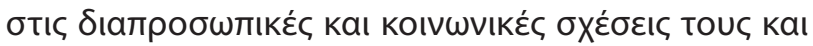

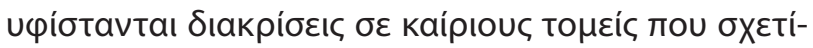

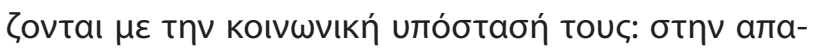

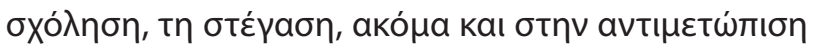

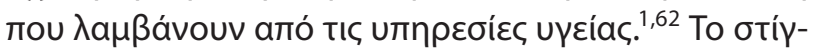

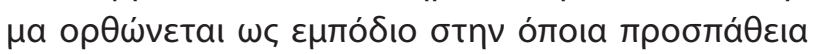

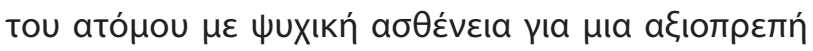

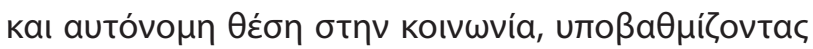

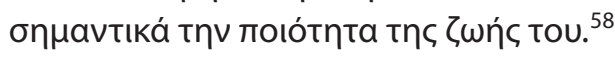




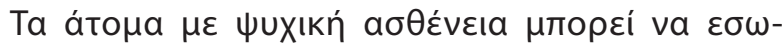

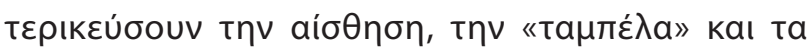

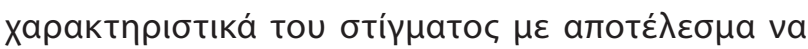

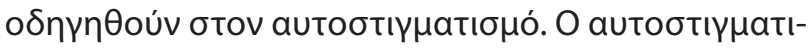

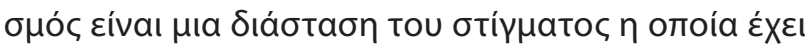

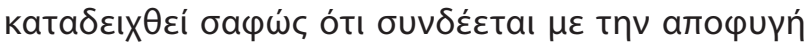

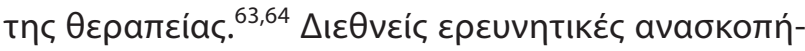

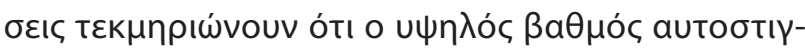

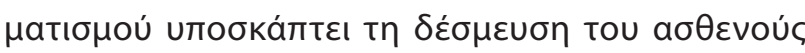

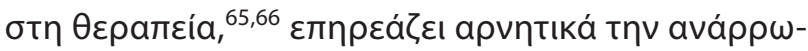

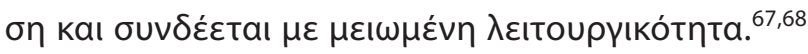

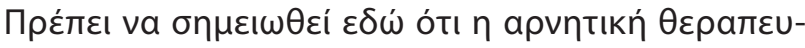

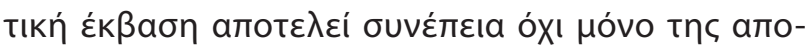

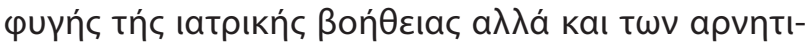

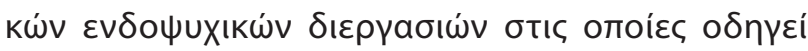

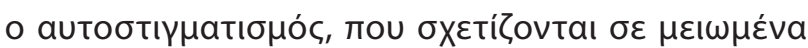

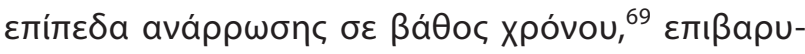

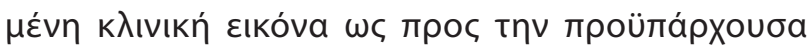

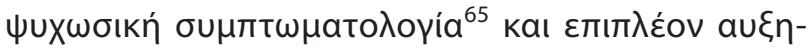

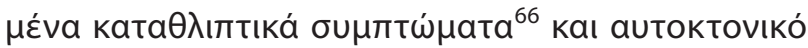

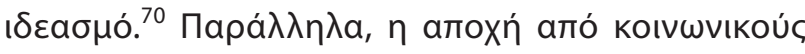

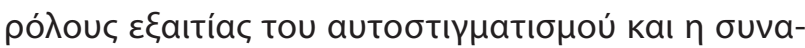

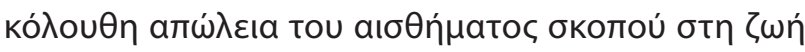

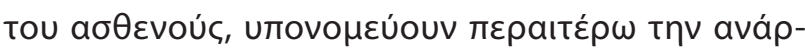
$\rho \omega \sigma n ́$ tou. ${ }^{71,72}$

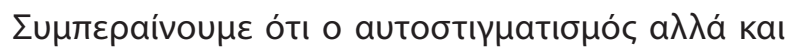

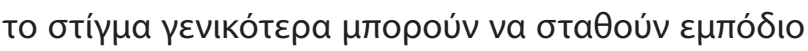

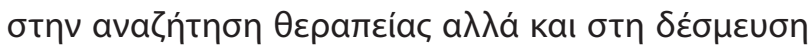

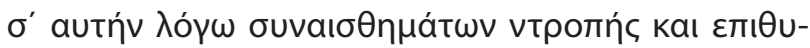

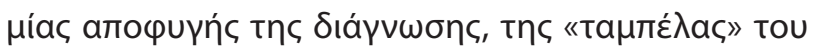

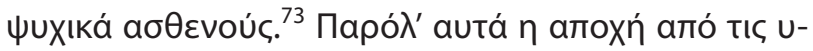

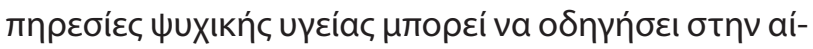

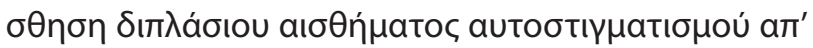

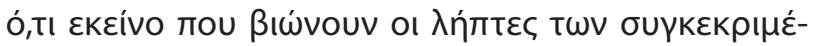

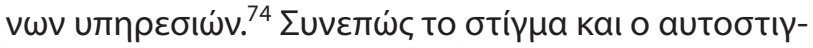

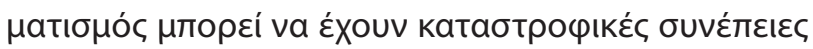

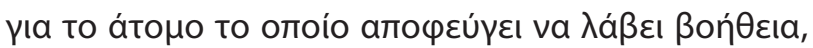

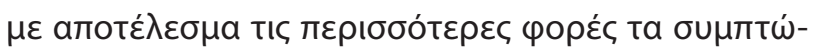

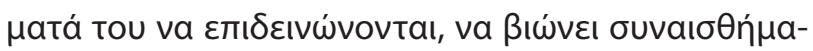

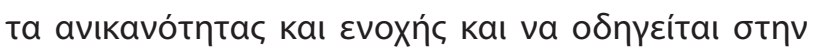

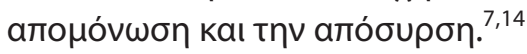

\section{Avtí ยmเกóyou}

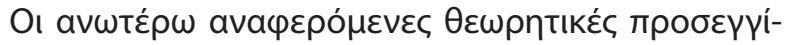

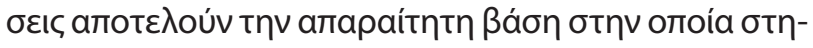

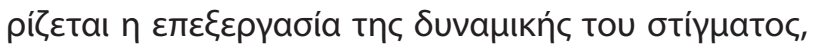

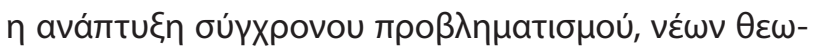

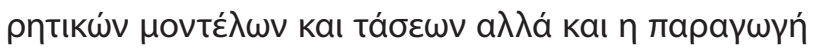

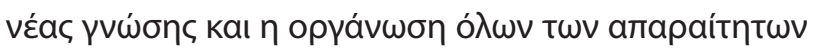

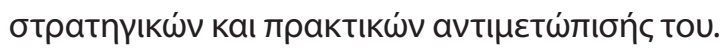

\title{
The stigma of mental illness: \\ A historical overview and conceptual approaches
}

\author{
M. Economou, ${ }^{1,2}$ A. Bechraki, ${ }^{2}$ M. Charitsi ${ }^{2}$ \\ ${ }^{1}$ First Department of Psychiatry, National and Kapodistrian University of Athens, Eginition Hospital, Athens, \\ 2University Mental Health, Neuroscience and Precision Medicine Research Institute "Costas Stefanis" (UMHRI), Athens, Greece
}

Psychiatriki 2020, 31:36-46

The present article explores the concept of stigma from a historical and theoretical perspective. At first, the conceptual origin of the term "stigma" is presented as well as its subsequent course and incorporation in the scientific field. The term stigma originates from ancient Greek language and in

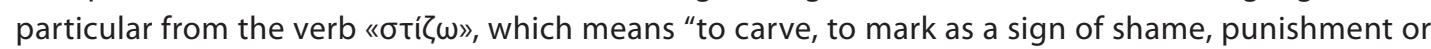
disgrace". In contemporary thinking about stigma, the work of Erving Goffman is seminal. According to him, stigmatization is elicited by the presence of a socially undesirable characteristic, which signals otherness. When this characteristic becomes conspicuous during a social interaction, it may act in a disqualifying manner for the identity of the person who bears it. One of the first theories on social stigma which attracted increased scientific attention is labeling theory by Thomas Scheff. Later 
on, the conceptual model of Corrigan and Watson underscored the main constituents of stigma, namely stereotypes, prejudices and discrimination; whereas the theoretical framework of Link and Phelan stressed labeling, stereotyping, separation, status loss and discrimination as interconnected components in a power situation. During the last half of the previous century, the concept of stigma came to the fore and gained growing research attention, especially due to shedding light on the ways whereby people with mental disorders were treated socially. Most of the literature has focused on recording the general population's level of knowledge and lay beliefs about mental illness as well as on exploring social attitudes and desired social distance from people with mental disorders. Converging evidence indicates that stereotypical beliefs and discriminatory attitudes against people with mental illness prevail worldwide; while illness severity, poor therapeutic outcome, disturbances in patients' emotional expression during a social interaction, incidents of violent or dangerous behaviours and labeling have all been shown to influence public stigma. Regarding lay respondents' correlates of public stigma; male gender, older age, lower socio-economic status, lower educational attainment and residence in semi-urban or rural areas have been linked to unfavourable attitudes towards people with mental disorders; while of outmost importance is personal experience/ familiarity with mental illness.

Key words: Stigma, mental illness, historical overview, conceptual approaches, stereotypes, prejudices, discrimination.

\section{BıßAtoypacpía}

1. Sartorius $\mathrm{N}$. One of the last obstacles to better mental health care: the stigma of mental illness. In: Guimón J, Fischer W, Sartorius N (eds) The image of madness. Karger, Basel, 1999

2. Burton R. The anatomy of melancholy. Faulkner TC, Kiessling NK, Blair RL (eds) Clarendon Press, Oxford, 1989

3. Lopez-lbor JJ. The WPA and the fight against stigma because of mental diseases. World Psychiatry 2002, 1:16-20, PMID: 16946818

4. Sartorius N, Schulze H. Reducing the stigma of mental illness: A report from a global programme of the World Psychiatric Association. Cambridge University Press, New York, 2005

5. Goffman E. Stigma: Notes on the Management of Spoiled Identity. Penguin, Harmondsworth, 1970

6. Scheff TJ. Being mentally ill: A sociological theory. Aldine, Chicago, 1966

7. Goffman E. Stigma. Penguin, London, 1963

8. Jones EE, Farina A, Hastorf AH, Markus H, Miller DT, Scott RA. Social Stigma: The Psychology of Marked Relationships. Freeman, New York, 1984

9. Corrigan PW, Watson AC. Understanding the impact of stigma on people with mental illness. World Psychiatry 2002, 1:16-20, PMID: 16946807

10. Link BG, Phelan JC. Conceptualizing stigma. Annu Rev Sociol 2001, 27:363-385, doi: 10.1146/annurev.soc.27.1.363

11. Weiner B. Judgments of responsibility: A foundation for a theory of social conduct. Guilford Press, New York, 1995

12. Corrigan PW. Mental health stigma as social attribution: Implications for research methods and attitude change. Clin Psychol Sci Pract 2000, 7:48-67, doi:10.1093/clipsy.7.1.48

13. Corrigan PW, River LP, Lundin RK, Uphoff Wasowski K, Campion $\mathrm{J}$, Mathisen $\mathrm{H}$ et al. Stigmatizing attributions about mental ill- ness. J Community Psychol 2000, 28:91-102, doi: 10.1002/ (SICI)1520-6629(200001)28:1<91::AID-JCOP9>3.0.CO;2-M

14. Corrigan PW, Watson AC, Heyrman M, Warpinski A, Gracia G, Slopen $\mathrm{N}$ et al. Structural stigma in state legislation. Psychiatr Serv 2005, 56:557-563, doi:10.1176/appi.ps.56.5.557

15. Phelan JC, Bromet EJ, Link BG. Psychiatric illness and family stigma. Schizophr Bull 1998, 24:115-126, doi: 10.1093/oxfordjournals.schbul.a033304

16. Persaud R. Psychiatrists suffer from stigma too. B J Psych Bull 2000, 24:284-285, doi: 10.1192/pb.24.8.284

17. Link BG, Phelan JC. Stigma and its public health implications. Lancet 2006, 367:528-529, doi: 10.1016/S0140-6736 (06)68184-1

18. Star S. The publics' ideas about mental illness. Paper presented at the Annual Meeting of the National Association for Mental Health, 5 November 1955. Indianapolis

19. Cumming E, Cumming G. Closed ranks: an experiment in mental health education. Harvard University Press, Cambridge, 1957

20. Whatley C. Social attitudes towards discharged patients. Soc Problems 1959, 6:313-320, doi: 10.2307/799364

21. Bogardus EM. Measuring social distance. J Appl Sociol 1925, 9: 299-308

22. Owen CA, Eisner HC, McFaul TR. A half century of social distance research: National replication of the Bogardus' studies. Sociol Soc Res 1981, 66:80-98

23. Angermeyer MC, Matschinger $\mathrm{H}$. Social distance towards the mentally ill: results of representative survey in the Federal republic of Germany. Psychol Med 1997, 27:131-141, doi: 10.1017/s0033291796004205

24. Angermeyer MC, Beck M, Matschinger H. Determinants of the public's preference for social distance from people with schizo- 
phrenia. Can J Psychiatry 2003, 48: 663-668, doi: 10.1177/ 070674370304801004

25. Crocetti G, Spiro JR, Siassi I. Are the ranks closed? Attitudinal social distance and mental illness. Am J Psychiatry 1971, 127: 1121-1127

26. Eker D. Attitudes toward mental illness: recognition, desired social distance, expected burden and negative influence on mental health among Turkish freshmen. Soc Psychiatry Psychiatr Epidemiol 1989, 24:146-150, doi: 10.1007/bf01788024

27. Brockington $\mathrm{I}$, Hall $\mathrm{P}$, Levings $\mathrm{J}$ et al. The community's tolerance of the mentally ill. Br J Psychiatry 1993, 162:93-99, doi: 10.1192/bjp.162.1.93

28. Madianos M, Economou M, Hatjiandreou $M$ et al. Changes in public attitudes towards mental illness in the Athens area (1979/1980-1994). Acta Psychiatr Scand 1999, 99:73-78, doi: 10.1111/j.1600-0447.1999.tb05387.x

29. Angermeyer MC, Dietrich S. Public beliefs about and attitudes towards people with mental illness: a review of population studies. Acta Psychiatr Scand 2006, 113:163-179, doi: 10.1111/j.1600-0447.2005.00699.x

30. Martin JK, Pescosolido BA, Tuch SA. Of fear and loathing: The role of "disturbing behavior", labels and causal attributions in shaping public attitudes toward people with mental illness. $J$ Health Soc Behav 2000, 41: 208-223, doi: 10.2307/2676306

31. Angermeyer MC, Matschinger H, Corrigan P. Familiarity with mental illness and social distance from people with schizophrenia and major depression: Testing a model using data from a representative population survey. Schizophr Res 2004, 69: 175-182, doi: 10.1016/s0920-9964(03)00186-5

32. Magliano L, Fiorillo A, de Rosa C et al. Beliefs about schizophrenia in Italy: a comparative nationwide survey of the general public, mental health professionals, and patients' relatives. Can J Psychiatry 2004, 49:322-330, doi: 10.1177/070674370404900508

33. Lauber $C$, Nordt $C$, Falcato $L$ et al. Factors influencing social distance toward people with mental illness. Commun Ment Health J 2004, 40:265-274, doi: 10.1023/B:COMH.00000269 99.87728.2d

34. Stuart H, Arboleda-Florez J. Community attitudes towards people with schizophrenia. Can J Psychiatry 2001, 46:245-252, doi: 10.1177/070674370104600304

35. Gaebel W, Baumann A, Witte AM et al. Public attitudes towards people with mental illness in two German cities. Results of a public survey under special considerations of schizophrenia. Eur Arch Psychiatry Clin Neurosci 2002, 252:278-287, doi: 10.1007/s00406-002-0393-2

36. Thompson A, Stuart H, Bland RC et al. Attitudes about schizophrenia from the pilot site of the WPA worldwide campaign against the stigma of schizophrenia. Soc Psychiatry Psychiatr Epidemiol 2002, 37:475-482, doi: 10.1007/s00127-002-0583-2

37. Economou M, Richardson C, Gramandani C, Stalikas A, Stefanis C. Knowledge about schizophrenia and attitudes towards people with schizophrenia in Greece. Int J Soc Psychiatry 2009, 55:361-371, doi: 10.1177/0020764008093957

38. Mouzas OD, Angelopoulos NV, Liakos A. Public opinions about mental illness in a Greek area: The influence of sociodemographic factors. Psychiatriki 2008, 19:337-349, PMID: 22218082
39. Arvanity A. Public attitudes towards mentally ill. Democritus University of Thrace 2008 (Cited 23 November 2019). Available from http://thesis.ekt.gr/thesisBook Reader/id/21014\#page/1/ mode/2up

40. Melissa C, Mavraki C, Gourni M, Tsalkanis A, Pilatis N, Argyriou $G$ et al. People's attitudes towards patients with mental illness in Greece. ICUs Nurs Web J 2006, 27:1-12

41. Tzouvara V, Papadopoulos C. Public stigma towards mental illness in the Greek culture. J Psychiatr Ment Health Nurs 2014, 21:931-938, doi: 10.1111/jpm.12146

42. Anagnostopoulos F, Hantzi A. Familiarity with and social distance from people with mental illness: Testing the mediating effects of prejudiced attitudes. J Community Appl Soc Psychol 2011, 21:451-460, doi: 10.1002/casp.1082

43. Saridi M, Kordosi A, Toska A, Peppou LE, Economou M, Souliotis K. Attitudes of health professionals towards the stigma surrounding depression in times of economic crisis. Int J Soc Psychiatry 2017, 63:115-125, doi: 10.1177/0020764016685348

44. Link BG, Cullen FT, Frank J, Wozniak JF. The social rejection of former mental patients: Understanding why labels matter. Am J Soc 1987, 92:1461-1500, doi: 10.1086/228672

45. Read J, Law A. The relationship of causal beliefs and contact with users of mental health services to attitudes to the 'mentally ill'. Int J Soc Psychiatry 1999, 45:216-229, doi: 10.1177/002076409904500309

46. Sartorius N, Schulze H. Reducing the stigma of mental illness: A report from a global programme of the World Psychiatric Association. Cambridge University Press, New York, 2005

47. Crisp AH, Gelder M, Rix S, Meltzer HI, Rowlands OJ. Stigmatization of people with mental illnesses. Br J Psychiatry 2000, 177:4-7, doi: 10.1192/bjp.177.1.4

48. Sugiura T, Sakamoto S, Tanaka E et al. Labeling effect of Seishin-bunretsu-byou, the Japanese translation for schizophrenia: an argument for relabeling. Int J Soc Psychiatry 2001, 47:43-51, doi: 10.1177/002076400104700204

49. Link BG, Phelan JC, Bresnahan M, Stueve A, Pescosolido BA. Public conceptions of mental illness: Labels causes, dangerousness and social distance. Am J Publ Health 1999, 89:13281333, doi: 10.2105/ajph.89.9.1328

50. Penn D, Guynan K, Daily T, Spaulding WD, Garbin CP, Sullivan $M$. Dispelling the stigma of schizophrenia: what sort of information is best? Schizophr Bull 1994, 20:567-578, doi: 10.1093/ schbul/20.3.567

51. Corrigan PW, Watson, AC. The stigma of psychiatric disorders and the gender, ethnicity, and education of the perceiver. Community Ment Health J 2007, 43:439-458, doi: 10.1007/ s10597-007-9084-9

52. Townley G, Brusilovskiy E, Salzer M. Urban and non-urban differences in community living and participation among individuals with serious mental illnesses. Soc Sci Med 2017, 177: 223-230, doi: 10.1016/j.socscimed.2017.01.058

53. Buizza C, Ghilardi A, Ferrari C. Beliefs and prejudices versus knowledge and awareness: how to cope with stigma against mental illness. A college staff e-survey. Community Ment Health J 2017, 53: 589-597, doi: 10.1007/s10597-017-0116-9 
54. Link BG, Cullen FT. Contact with the mentally ill and perceptions of how dangerous they are. J Health Soc Behav 1986, 27:289-302, PMID: 3559124

55. Markovitz FA, Engelman DJ. The "Own" and the "Wise": Does stigma status buffer or exacerbate social rejection of college students with a mental illness? Deviant Behav 2017, 38:744-755, doi: 10.1080/01639625.2016.1197673

56. Zen G, Lepri B, Ricci E, Lanz O. Space speaks: towards socially and personality aware visual surveillance. Paper presented at 1st ACM International Workshop on Multimodal Pervasive Video Analysis (MPVA 2010), 29 October 2010. Firenze, MPVA'10 Proceedings of the 2010 ACM Workshop on Multimodal Pervasive Video Analysis, pp. 37-42, doi: 10.1145/1878039.1878048

57. Schulze B, Angermeyer MC. Subjective experiences of stigma. A focus group study of schizophrenic patients, their relatives and mental health professionals. Soc Sci Med 2003, 56:299312, doi: 10.1016/s0277-9536(02)00028-x

58. Gaebel W, Zäske H, Bauman AE. The relationship between mental illness severity and stigma. Acta Psychiatr Scand Suppl 2006, 113:41-45, doi: 10.1111/j.1600-0447.2005.00716.x

59. Bauman AE, Craige E, Zäske $H$ et al. Interpersonal factors contributing to the desire for social distance. Paper presented at the XIIIth World Congress of Psychiatry, 10-15 September 2005. Cairo

60. Grausgruber A, Meise U, Katsching H, Schöny W, Fleischhacker WW. Patterns of social distance towards people suffering from schizophrenia in Austria: a comparison between the general public, relatives and mental health staff. Acta Psychiatr Scan 2007, 115:310-319, doi: 10.1111/j.1600-0447.2006.00882.x

61. Crisp AH, Gelder M, Rix S, Meltzer HI, Rowlands OJ. Stigmatization of people with mental illnesses. Br J Psychiatry 2000, 177:4-7, doi: 10.1192/bjp.177.1.4

62. Marwaha S, Johnson S. Schizophrenia and employment: A review. Soc Psychiatry Psychiatr Epidemiol 2004, 39:337-349, doi: 10.1007/s00127-004-0762-4

63. Barney LJ, Griffiths KM, Jorm A, Christensen H. Stigma about depression and its impact on help-seeking intentions. Aust N Z J Psychiatry 2006, 40: 51-54, doi: 10.1080/j.1440-1614. 2006.01741.x

64. Clement S, Schauman O, Graham T, Maggioni F, Evans-Lacko $\mathrm{S}$, Bezborodovs $\mathrm{N}$ et al. What is the impact of mental health stigma on help-seeking? A systematic review of quantitative and qualitative studies. Psychol Med 2015, 45:11-27, doi: 10.1017/S0033291714000129

65. Livingston JD, Boyd JE. Correlates and consequences of internalized stigma for people living with mental illness: a systematic review and meta-analysis. Soc Sci Med 2010, 71: 2150-2161, doi: 10.1016/j.socscimed.2010.09.030

66. Boyd JE, Adler EP, Otiligam PG, Peters T. Internalized stigma of mental illness (ISMI) Scale: a multinational review. Compr Psychiatry 2014, 55: 221-231, doi: 10.1016/j.comppsych. 2013.06.005

67. Yanos PT, Lysaker PH, Roe D. Internalized stigma as a barrier to improvement in vocational functioning among people with schizophrenia-spectrum disorders. Psychiatry Res 2010 (a), 178: 211-213, doi: 10.1016/j.psychres.2010.01.003

68. Yanos PT, Lysaker PH, Roe D. The impact of illness identity on recovery from severe mental illness. Am J Psychiatr Rehabil 2010(b), 13:73-93, doi: 10.1080/15487761003756860

69. Oexle N, Muller M, Kawohl W, Xu Z, Viering S, Wyss C et al. Self-stigma as a barrier to recovery: a longitudinal study. Eur Arch Psychiatry Clin Neurosci 2018, 268:209-212, doi: 10.1007/ s00406-017-0773-2

70. Oexle N, Rusch N, Viering S, Wysss C, Seifritz E. Self-stigma and suicidality: a longitudinal study. Eur Arch Psychiatry Clin Neurosci 2017, 267: 359-361, doi: 10.1007/s00406-016-0698-1

71. Yanos PT, Roe D, Markus K, Lysaker PH. Pathways between internalized stigma and outcomes related to recovery in schizophrenia spectrum disorders. Psychiatr Serv 2008, 59:1437-1442, doi: 10.1176/appi.ps.59.12.1437

72. Munoz M, Sanz M, Perez-Santos E, Quiroga MA. Proposal of a socio-cognitive-behavioral structural equation model of internalized stigma in people with severe and persistent mental illness. Psychiatr Res 2011, 186: 402-408, doi: 10.1016/j.psychres. 2010.06.019

73. Corrigan PW. How clinical diagnosis might exacerbate the stigma of mental illness. Soc Work 2007, 52:31-39, doi: 10.1093/ $\mathrm{sw} / 52.1 .31$

74. Zartaloudi A, Madianos M. Stigma related to help-seeking from a mental health professional. Health Sci J 2010, 4:77-83

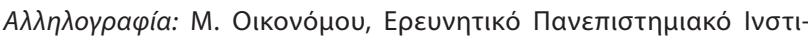

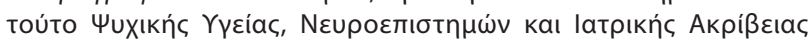

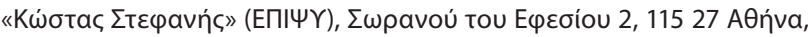
$\operatorname{T\eta } \lambda:$ 210-61 70 071-2, 6170 804-5, Fax: 210-65 64021

e-mail: antistigma@epipsi.eu, marinaeconomou1@gmail.com 